\title{
Radiotherapy-exposed CD8+ and CD4+ neoantigens enhance tumor control
}

\author{
Claire Lhuillier, ${ }^{1}$ Nils-Petter Rudqvist, ${ }^{1}$ Takahiro Yamazaki, ${ }^{1}$ Tuo Zhang, ${ }^{2}$ Maud Charpentier, ${ }^{1}$ Lorenzo Galluzzi, ${ }^{1,3,4}$ \\ Noah Dephoure, ${ }^{3,5}$ Cristina C. Clement, ${ }^{1}$ Laura Santambrogio, ${ }^{1,4}$ Xi Kathy Zhou, ${ }^{6}$ Silvia C. Formenti, ${ }^{1,3,7}$ and Sandra Demaria ${ }^{1,3,8}$ \\ 'Department of Radiation Oncology and ${ }^{2}$ Department of Microbiology and Immunology, Weill Cornell Medicine, New York, New York, USA. ${ }^{3}$ Sandra and Edward Meyer Cancer Center, New York, New York, \\ USA. ${ }^{4}$ Caryl and Israel Englander Institute for Precision Medicine, New York, New York, USA. ${ }^{5}$ Department of Biochemistry, ${ }^{6}$ Division of Biostatistics and Epidemiology, Department of Healthcare Policy and \\ Research, and ${ }^{7}$ Department of Medicine, Weill Cornell Medicine, New York, New York, USA. ${ }^{8}$ Department of Pathology and Laboratory Medicine, Weill Cornell Medical College, New York, New York, USA.
}

\begin{abstract}
Neoantigens generated by somatic nonsynonymous mutations are key targets of tumor-specific T cells, but only a small number of mutations predicted to be immunogenic are presented by MHC molecules on cancer cells. Vaccination studies in mice and patients have shown that the majority of neoepitopes that elicit $T$ cell responses fail to induce significant antitumor activity, for incompletely understood reasons. We report that radiotherapy upregulates the expression of genes containing immunogenic mutations in a poorly immunogenic mouse model of triple-negative breast cancer. Vaccination with neoepitopes encoded by these genes elicited $\mathrm{CD8}^{+}$and $\mathrm{CD4} 4^{+} \mathrm{T}$ cells that, whereas ineffective in preventing tumor growth, improved the therapeutic efficacy of radiotherapy. Mechanistically, neoantigen-specific CD8 ${ }^{+} \mathrm{T}$ cells preferentially killed irradiated tumor cells. Neoantigen-specific CD4 ${ }^{+} \mathrm{T}$ cells were required for the therapeutic efficacy of vaccination and acted by producing Th1 cytokines, killing irradiated tumor cells, and promoting epitope spread. Such a cytotoxic activity relied on the ability of radiation to upregulate class II MHC molecules as well as the death receptors FAS/CD95 and DR5 on the surface of tumor cells. These results provide proof-of-principle evidence that radiotherapy works in concert with neoantigen vaccination to improve tumor control.
\end{abstract}

\section{Introduction}

Mutation-associated neoantigens drive responses to immune checkpoint blockade (ICB) therapy in tumors with high mutational burden, including melanoma, non-small cell lung cancer (NSCLC), and mismatch repair-deficient cancers (1-4). Several clinical trials in melanoma and glioblastoma patients have demonstrated the feasibility, safety, and tolerability of neoantigen vaccines (5-8). Although these studies reported the induction of neoepitope-specific $\mathrm{T}$ cell responses in the vaccinated patients, the clinical benefit was overall modest. It is becoming clear that ameliorating the therapeutic efficacy of neoantigen vaccines requires interventions that can overcome immunosuppression in the tumor microenvironment. In addition, it also requires the optimization of neoantigen presentation to the immune system $(9,10)$.

\footnotetext{
Conflict of interest: SCF has received advisory or speaker compensation from AstraZeneca, Sanofi, EMD Serono, and Elekta, and research support from Bristol Myers Squibb, Merck, Eisai, Regeneron, Varian, and Janssen. SCF's daughter is an employee of Pfizer. SD has received compensation for consultant/advisory services from Lytix Biopharma, Mersana Therapeutics, EMD Serono, and Ono Pharmaceutical, and research support from Lytix Biopharma and Nanobiotix. LG has received consulting fees from OmniSEQ, AstraZeneca, Inzen, and the Luke Heller TECPR2 Foundation, and he is member of the Scientific Advisory Committee of Boehringer Ingelheim, The Longevity Labs, and OmniSEQ.

Copyright: () 2021, American Society for Clinical Investigation.

Submitted: April 7, 2020; Accepted: January 13, 2021; Published: March 1, 2021.

Reference information: J Clin Invest. 2021;131(5):e138740.

https://doi.org/10.1172/JCl138740.
}

Existing preclinical data indicate that even the most immunogenic neoepitopes elicit $\mathrm{T}$ cell responses unable to cause tumor rejection and that this is due, at least in part, to lack of presentation of the neoepitopes by MHC molecules on the cancer cells (11, 12). One of the most important factors in determining how well an antigen is presented by class I MHC (MHC-I) molecules is the relative rate of antigen synthesis and degradation, as well as its overall abundance $(13,14)$. Thus, we hypothesized that an intervention that increases the expression of a mutated gene could work in concert with neoantigen vaccination. Radiation elicits an acute transcriptional response with upregulated expression of DNA damage response genes, which are often mutated in cancer (15). Radiation also elicits multiple pathways involved in response to cellular stress and inflammation, including type I interferon signaling (1618). In support of the hypothesis that the radiation-upregulated proteome is displayed by MHC-I on irradiated cells, Reits and colleagues identified peptides derived from proteins involved in DNA repair and in protein breakdown in the MHC-I immunopeptidome of irradiated melanoma cells (17). We found that tumor-specific $\mathrm{CD}^{+} \mathrm{T}$ cells expanded in a metastatic NSCLC patient with complete response to focal radiotherapy and ICB were directed against an immunogenic mutation in a gene upregulated by radiation (19). These data support testing radiotherapy as an intervention that increases the expression of mutated genes to improve responses to a personalized neoantigen vaccination strategy (20).

Here we used $4 \mathrm{~T} 1$ cells, a mouse model of poorly immunogenic triple-negative breast cancer that possesses 293 somatic mutations, to test whether radiotherapy can increase the expres- 
sion of genes encoding immunogenic mutations. We identified two MHC-I and one MHC-II immunogenic neoepitopes encoded by 3 genes upregulated by radiotherapy, namely DExH-box helicase 58 (Dhx58; a RIG-I-like receptor also known as LGP2), cullin associated and neddylation dissociated 1 (Cand1), and adhesion G protein-coupled receptor F5 (Adgrf5). Vaccination with these 3 neoepitopes elicited polyfunctional $\mathrm{T}$ cell responses as well as CAND1-directed $\mathrm{CD}^{+}$cytotoxic $\mathrm{T}$ cells, but did not prevent $4 \mathrm{~T} 1$ tumor growth. However, vaccination was effective at inhibiting the growth of irradiated tumors, and $\mathrm{T}$ cells from vaccinated mice preferentially killed irradiated $4 \mathrm{~T} 1$ cells in vitro. The MHCII-restricted neoepitope was required for the therapeutic efficacy of the vaccine. Mechanistically, neoantigen-specific CD $4^{+} \mathrm{T}$ cells produced high levels of IFN- $\gamma$ and displayed cytotoxic activity against irradiated $4 \mathrm{~T} 1$ cells, which expressed low but detectable MHC-II and upregulated the cell surface death receptor FAS (also known as APO-1 or CD95) and DR5 (also known as TRAIL-R2). Overall, our study provides proof-of-principle evidence that radiotherapy can expose existing neoantigens to the immune system and that both $\mathrm{CD}^{+}$and $\mathrm{CD}^{+}$neoantigen-specific $\mathrm{T}$ cells are required for the antitumor activity in a poorly immunogenic model of breast cancer.

\section{Results}

Identification of $\mathrm{CD}^{+}$neoepitopes upregulated by radiation in $4 T 1$ cancer cells. For neoantigen identification, we first performed whole-exome and RNA sequencing of $4 \mathrm{~T} 1$ cells in vitro. We identified 224 genes containing a total of 293 somatic mutations, among which 154 mutations encoded by 115 genes were expressed at the RNA level (Figure 1A). Among them, 97 mutations passed additional filtering based on variant allele frequency. Expression of 11 mutated genes (corresponding to 13 mutations) was confirmed in vivo by whole-exome/RNA sequencing of DNA/RNA extracted from $4 \mathrm{~T} 1$ tumors growing in syngeneic BALB/c mice.

Next, to test the hypothesis that radiation increases the expression of genes encoding immunogenic mutations, we compared the expression of these 11 mutated genes in irradiated versus untreated $4 \mathrm{~T} 1$ cells. Cells were irradiated with a dose of 8 Gy on 3 consecutive days, selected because this dose and fractionation achieved the best synergy with ICB in preclinical studies (21), and similar radiation doses were effective at increasing responses to ICB in cancer patients $(19,22)$. Four genes, Adgrf5, Cand1, Dhx58, and retinoic acid early transcript $1 \mathrm{E}$ (Raet1e), containing 5 mutations, were significantly upregulated by radiation (Figure 1B). Using the NetMHC algorithm and the pVAC-Seq neoantigen discovery pipeline, 9 neoepitopes from these 4 genes were predicted to bind MHC-I proteins with an affinity less than $300 \mathrm{nM}$, and 1 neoepitope for each mutation was chosen for testing based on the highest affinity score (Table 1). Binding of the corresponding peptides to MHC-I molecules was tested in stabilization assays using RMA-S cells expressing the mouse MHC-I allele $\mathrm{H} 2-\mathrm{K}^{\mathrm{d}}$ or $\mathrm{H} 2-\mathrm{L}^{\mathrm{d}}$ (Figure 1C). The CAND1-derived peptide (hereafter CAND1) showed a strong binding to $\mathrm{H} 2-\mathrm{K}^{\mathrm{d}}$, comparable to that of the influenza epitope HA515, used as positive control. The peptide derived from ADGRF5 (hereafter, ADGRF5) also bound H2-K but to a lower extent. For H2-L $\mathrm{L}^{\mathrm{d}}, 2$ peptides derived from RAET1E and DHX58 (hereafter RAET1E and DHX58, respectively) showed strong binding, comparable to that of the positive control, AH1-A5 (an optimized ligand for $\mathrm{H} 2-\mathrm{L}^{\mathrm{d}}$; ref. 23). Another peptide derived from a second mutated sequence of RAET1E was toxic and was not further tested. Since the immunogenicity of a peptide is significantly correlated with the stability of the MHC-peptide complex (24), the latter was monitored, revealing that the most stable complexes were formed by CAND1/H2-K $\mathrm{K}^{\mathrm{d}}$ and DHX58/H2- $\mathrm{L}^{\mathrm{d}}$ (half-life $>6$ hours) whereas ADGRF5/H2-K ${ }^{\mathrm{d}}$ and RAET1E/H2- $\mathrm{L}^{\mathrm{d}}$ complexes were short-lived (half-life $<6$ hours; Figure 1D).

In vivo immunogenicity of neoepitopes upregulated by radiation. To test the immunogenicity of $\mathrm{CD}^{+}$neoepitopes upregulated by radiation, naive $\mathrm{BALB} / \mathrm{c}$ mice were vaccinated with 2 peptides binding to different $\mathrm{MHC}$ proteins to avoid competition. Among MHC-I binders, we included as controls 2 neoepitopes derived from a mutant protein encoded by leukotriene A4 hydrolase (Lta $4 h$ ), which was not upregulated by radiation (Supplemental Figure 1A; supplemental material available online with this article; https://doi.org/10.1172/JCI138740DS1). Binding of 1 of the 2 peptides, LTA4H-a, to $\mathrm{H} 2-\mathrm{K}^{\mathrm{d}}$ was comparable to that of the positive control HA515, while the other, LTA4H-b, showed slightly lower binding (Supplemental Figure 1B and Table 1). In addition, we tested the immunogenicity of 2 MHC-II neoepitopes. One is encoded by mutant ADAM metallopeptidase with thrombospondin type 1 motif 9 (Adamts9), and was previously predicted by Kreiter et al. (25) to bind to MHC-II and to be a CD4 ${ }^{+}$neoepitope. We also searched for predicted MHC-II neoepitopes in the mutated genes upregulated by radiation and identified a mutated peptide in Adgrf5 as a potential candidate (Table 1).

Mice were immunized twice, 1 week apart, with adjuvant alone or in combination with peptides (Figure 2A). One week after the second immunization, cell suspensions from vaccine-draining lymph nodes and spleen were restimulated ex vivo with the corresponding neoepitopes, followed by intracellular staining of $\mathrm{CD}^{+}$ and $\mathrm{CD}^{+} \mathrm{T}$ cells for IFN- $\gamma$ and TNF- $\alpha, 2$ cytokines involved in $\mathrm{T}$ cell effector functions. Among the $\mathrm{CD}^{+}$neoepitopes, ADGRF5, RAET1E, LTA4H-a, and LTA4H-b did not elicit a response above the background seen in mice vaccinated with adjuvant alone (Figure 2B). In contrast, DHX58 and CAND1 stimulated a polyfunctional $\mathrm{CD}^{+} \mathrm{T}$ cell response with most responding cells producing both IFN- $\gamma$ and TNF- $\alpha$ (Figure 2, B and C). Among CD4 ${ }^{+}$neoepitopes, only the ADGRF5-derived peptide (hereafter ADGRF5-II) stimulated the production of IFN- $\gamma$ and TNF- $\alpha$ by CD $4^{+} \mathrm{T}$ cells, indicating that it was able to prime $\mathrm{T}$ cell responses in vivo (Figure 2, D and E). Thus, although the mutation encoded by Adgrf5 did not stimulate a CD8 ${ }^{+} \mathrm{T}$ cell response, it was a good target for $\mathrm{CD} 4^{+} \mathrm{T}$ cells. IFN- $\gamma$ production was further confirmed in the supernatants of cells from draining lymph nodes after CAND1 and ADGRF5-II restimulation (Supplemental Figure 2A). Additional cytokine profiling of ADGRF5-II-stimulated CD $4^{+} \mathrm{T}$ cells confirmed that they produce IFN- $\gamma$ but not IL-4, IL-5, IL-10, and IL-13, indicating a Th1 phenotype (Supplemental Figure 2, B-E).

Comparison of the binding ability of the mutated and nonmutated (WT) peptides to the respective MHC-I alleles showed a large differential for CAND1 $\left(\mathrm{EC}_{50}=1.5 \mu \mathrm{M}\right.$ and $58.4 \mu \mathrm{M}$, respectively), while there was no significant difference for DHX58 $\left(\mathrm{EC}_{50}\right.$ $=27.0 \mu \mathrm{M}$ and $29.2 \mu \mathrm{M}$, respectively; Figure $2 \mathrm{~F}$ ). $\mathrm{CD}^{+} \mathrm{T}$ cells from mice vaccinated with the CAND1 neoepitope did not react against 
A

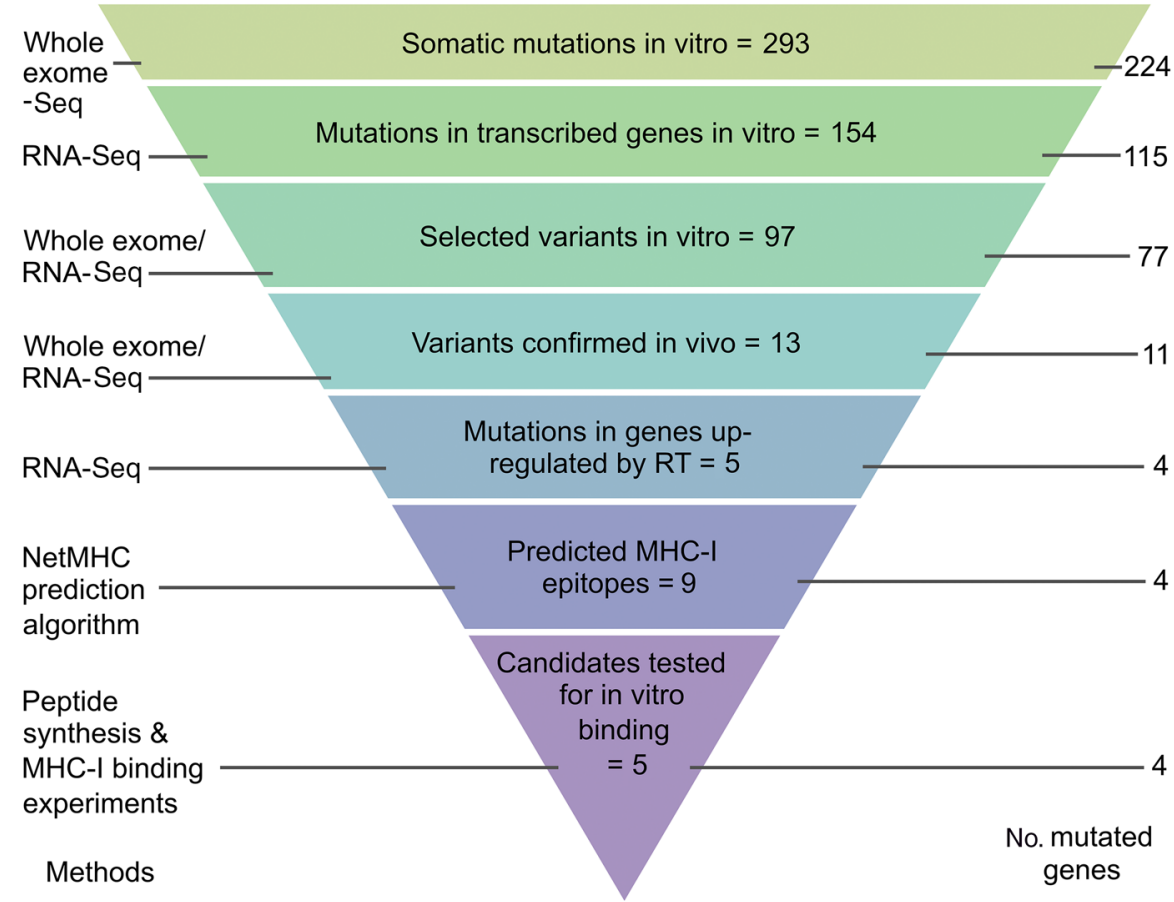

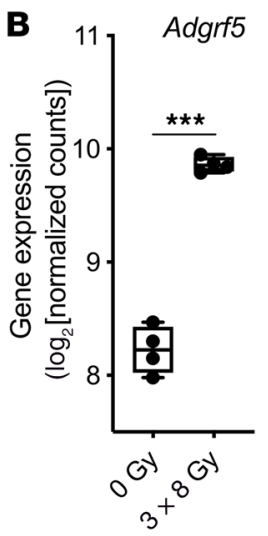
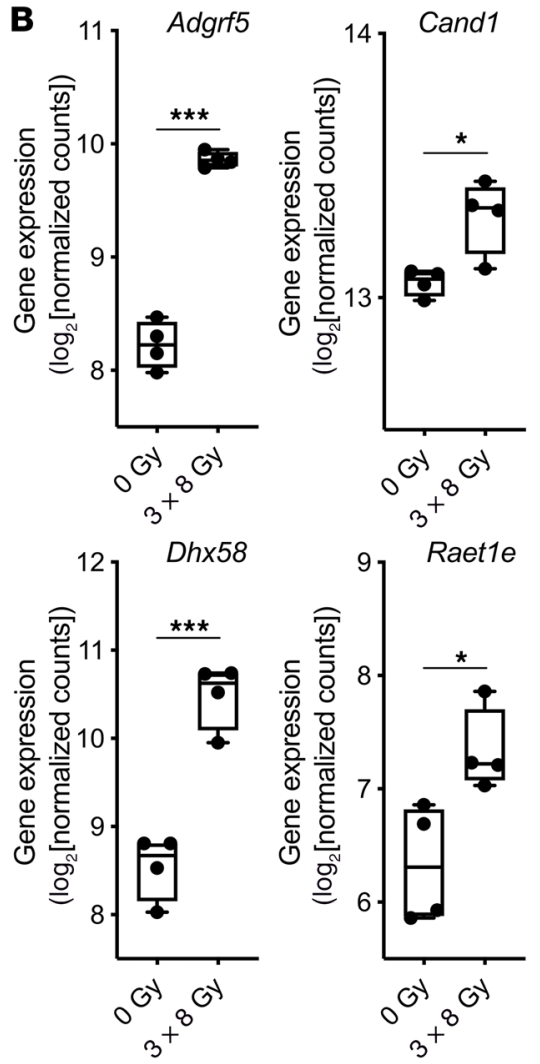
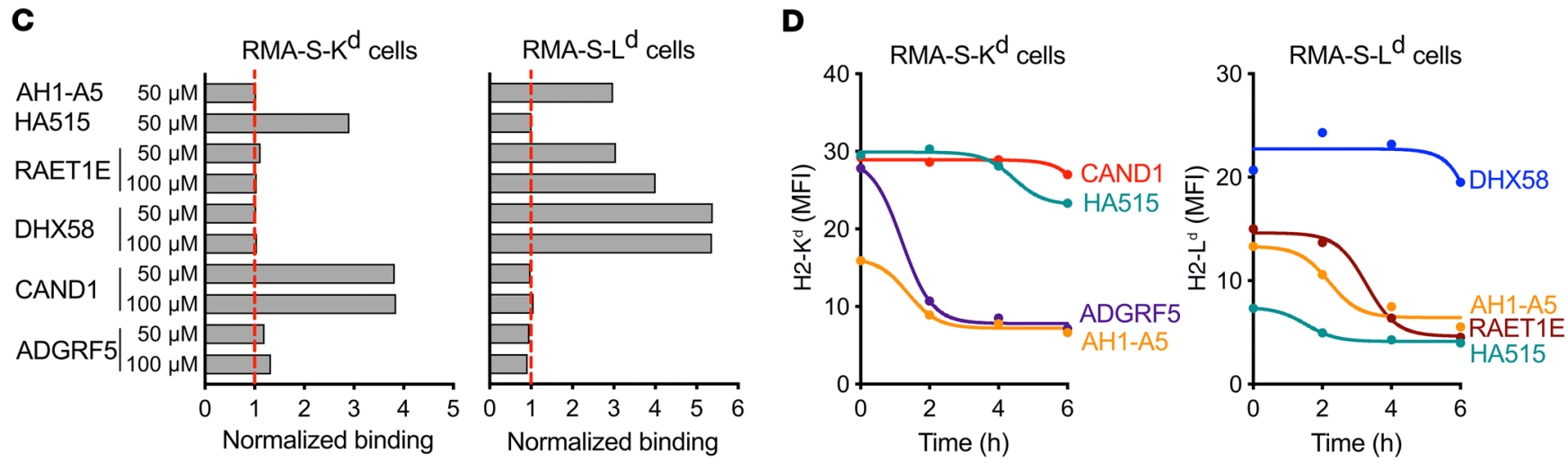

Figure 1. Prediction and in vitro validation of MHC-I neoepitopes upregulated by radiotherapy in $4 \mathrm{T1}$ breast cancer cells. (A) Tumor neoantigen identification pipeline. Whole-exome and RNA sequencing data were used to identify mutations in genes expressed by $4 \mathrm{~T} 1$ cells in vitro and in vivo and significantly upregulated by radiotherapy (RT). Candidate MHC-I binders were predicted using NetMHC and tested in binding assays. (B) RNA expression for Adgrf5, Dhx58, Cand1, and Raet1e genes as determined by RNA sequencing of $4 \mathrm{T1}$ cells irradiated ( $3 \times 8$ Gy) or untreated ( 0 Gy) in vitro ( $n=4$ independent experiments). Data are expressed as mean \pm SEM of normalized counts (log transformed). Comparisons between untreated and irradiated samples were made with unpaired 2-tailed Welch's $t$ test; ${ }^{*} P<0.05$, ${ }^{* *} P<0.001$. (C) MHC-I binding assay. RMA-S cells expressing either $\mathrm{H} 2-\mathrm{K}^{\mathrm{d}}$ (left) or H2-L ${ }^{\mathrm{d}}$ (right) were incubated with candidate peptides $(50$ or $100 \mu \mathrm{M})$ for 2 hours and then tested for MHC-I expression by flow cytometry. The binding capability of each peptide was calculated by normalization of the mean fluorescence intensity (MFI) of H2- $\mathrm{K}^{\mathrm{d}}$ or H2- $\mathrm{L}^{\mathrm{d}}$ in the presence of peptides compared with the MFI in the absence of peptides. HA515 peptide was used as positive control for $\mathrm{H2}-\mathrm{K}^{\mathrm{d}}$, whereas AH1-A5 was used as positive control for $\mathrm{H2}$ - $\mathrm{L}^{\mathrm{d}}$. (D) Stability assay over time. RMA-S cells were loaded with peptides at $26^{\circ} \mathrm{C}$ for 1 hour and then transferred to $37^{\circ} \mathrm{C}$ before determination of H2- $\mathrm{K}^{\mathrm{d}}$ (left) or H2- $\mathrm{L}^{\mathrm{d}}$ (right) $\mathrm{MFI}$ by flow cytometry at the indicated times.

the WT sequence (Supplemental Figure 2, F and G). Despite the similar binding of mutated and WT DHX58 to H2-L $\mathrm{L}^{\mathrm{d}}, \mathrm{CD} 8^{+} \mathrm{T}$ cells reacted significantly better against the mutated than against the WT peptide (Supplemental Figure $2 \mathrm{H}$ ). $\mathrm{CD} 4^{+} \mathrm{T}$ cells primed in vivo against the ADGRF5-II neoepitope also reacted, but to a lesser degree, against the WT epitope (Supplemental Figure 2I).
The CAND1 neoepitope is a target for $C D 8^{+}$cytotoxic $T$ cells. To determine whether vaccination with our 3 immunogenic neoepitopes (DHX58, CAND1, and ADGRF5-II) elicited antigen-specific cytotoxic $\mathrm{T}$ cells in vivo, mice were vaccinated twice with adjuvant alone (control) or with a cocktail of the 3 neoepitopes (Neo-vax) followed by intravenous injection of CFSE-labeled target cells in 
Table 1. Characteristics of candidate neoepitopes selected for in vitro and in vivo assays

\begin{tabular}{|c|c|c|c|c|c|c|c|}
\hline \multirow[t]{2}{*}{ Mutation type } & \multirow[t]{2}{*}{ Neoepitope } & \multirow[t]{2}{*}{ Allele } & \multirow[t]{2}{*}{ Length } & \multicolumn{2}{|c|}{ Amino acid sequences ${ }^{A}$} & \multicolumn{2}{|c|}{ Predicted affinity (nM) } \\
\hline & & & & Mutated & WT & Mutated & WT \\
\hline \multicolumn{8}{|c|}{ MHC-I-predicted epitopes } \\
\hline Missense V/M & ADGRF5 & $\mathrm{H} 2-\mathrm{K}^{\mathrm{d}}$ & 9 & PMFSMSSPI & PVESSMSSPI & 126 & 326 \\
\hline Missense R/L & DHX58 & $\mathrm{H} 2-\mathrm{L}^{\mathrm{d}}$ & 11 & LVPFSIPVFDI & RVPFSIPVFDI & 116 & 122 \\
\hline Missense V/I & LTA4H-a & $\mathrm{H} 2-\mathrm{K}^{\mathrm{d}}$ & 9 & SYQSITTDD & SYQSVITDD & 198 & 118 \\
\hline Missense $\mathrm{T} / \mathrm{l}$ & RAET1E & $\mathrm{H} 2-\mathrm{L}^{\mathrm{d}}$ & 11 & QIDSATWEFNI & QTPSATWEFNI & 99 & 374 \\
\hline \multicolumn{8}{|c|}{ MHC-II-predicted epitopes } \\
\hline Missense I/L & ADAMTS9 & $H 2-I^{d}$ & 15 & QKLERLDLTSMQQIITT & QKIRLDLTSMQIIITT & 357 & 305 \\
\hline Missense V/M & ADGRF5-II & $\mathrm{H} 2-\mathrm{IA}^{\mathrm{d}}$ & 15 & STPMFSMSSPISRRF & STPVFSMSSPISRRF & 162 & 238 \\
\hline
\end{tabular}

AMutated amino acid is underlined. ${ }^{\mathrm{B} T h i s ~ p e p t i d e ~ w a s ~ t o x i c ~ t o ~ t h e ~ c e l l s . ~}$

order to monitor specific killing in vivo. Target cells consisted of splenocytes isolated from a naive mouse, pulsed with each individual peptide (DHX58, CAND1, or ADGRF5-II) or with all 3 and labeled with a high concentration of CFSE (CFSEhi), mixed with unpulsed target cells labeled with a low concentration of CFSE $\left(\mathrm{CFSE}^{\mathrm{lo}}\right)$ at a 1:1 ratio. Selective elimination of the peptide-pulsed target cells was measured 24 hours later in the lymph nodes and spleen by flow cytometry analysis, as previously described (26, 27). Mice vaccinated with the HA515 peptide were used as a positive control for the assay and showed high in vivo cytotoxic activity against HA515-loaded target cells (Figure 3A). In mice vaccinated with Neo-vax, in vivo cytotoxicity was significant against the cells loaded with all 3 peptides and with CAND1 alone in both the spleen and the lymph nodes, indicating that this neoepitope was a main target of cytotoxic T cells (Figure 3, B and C). Intriguingly, there was no detectable cytotoxicity against the DHX58 neoepitope. Likewise, there was no significant cytotoxicity against the target cells loaded with ADGRF5-II in vivo or in vitro (Figure 3, C and D). In contrast, lymph node-derived $\mathrm{T}$ cells from Neo-vax-treated mice readily killed target cells pulsed with the CAND1-mutated but not the WT epitope in vitro, and the cytotoxicity was abrogated in the presence of MHC-I-blocking antibodies, confirming the specificity of $\mathrm{CD}^{+} \mathrm{T}$ cells for the mutated peptide presented by MHC-I (Figure 3D).

Next, T cells isolated from Neo-vax or adjuvant-only-vaccinated (Control) mice were incubated with untreated and irradiated 4T1 target cells labeled with high and low CFSE concentration, respectively (Figure 3E). Selective killing of irradiated as compared with nonirradiated $4 \mathrm{~T} 1$ cells was seen after coculture with lymph node cells from Neo-vax but not control mice (Figure 3, $\mathrm{F}$ and $\mathrm{G})$. Overall, these data demonstrate that vaccination with Neo-vax elicits CD8 ${ }^{+}$cytotoxic T cells specific for the CAND1 neoantigen that can selectively kill irradiated $4 \mathrm{~T} 1$ cells.

Increased presentation of CAND1 and ADGRF5-II neoepitopes on irradiated cancer cells. To confirm that radiotherapy enhanced the presentation of the identified neoepitopes on MHC molecules at the surface of $4 \mathrm{~T} 1$ cells, we performed mass spectrometry-based (MS-based) immunopeptidomics coupled with selected reaction monitoring (SRM) quantitation analysis (28). MHC-I and MHC-II complexes were immunopurified from untreated and irradiated $4 \mathrm{~T} 1$ cells, and after elution of the MHC-bound peptides, the samples were spiked with heavy isotope-labeled standard peptides for further quantitation by targeted SRM MS analysis (Figure 4 and Supplemental Table 1). The control AH1 peptide was identified and quantified with a slight increase in the copy number/ single cell in the irradiated $4 \mathrm{~T} 1$ cells (103.9) as compared with the untreated cells (80.4; Figure 4A and Table 2). In contrast, CAND1 was expressed at very low copy number/single cell in the untreated cells (23.3) but had a markedly increased abundance after irradiation (134.5), comparable to the AH1 peptide (Figure 4B and Table 2). We could not measure changes in DHX58 as it was undetectable in our samples, likely reflecting a very low presentation on the surface of $4 \mathrm{~T} 1$ cells. Despite a barely detectable expression of MHC-II molecules on untreated 4T1 cells, the MHC-II-bound ADGRF5-II peptide was identified by targeted MS analysis, having at least 3-fold increase in the copy number/single cell in the irradiated 4T1 cells (Figure 4C and Table 2). Consistently, radiation increased MHC-II expression on 4T1 cells (Supplemental Figure 4A). The sequence of CAND1 and ADGRF5-II peptides was further confirmed by MS2 profiling (Supplemental Figures 3 and 4, Supplemental Table 2, and Supplemental Methods).

Vaccination with radiation-upregulated neoantigens improves tumor control by radiotherapy. To investigate the role of the identified neoantigens in antitumor immune responses, mice were vaccinated twice with Neo-vax or adjuvant alone before 4T1 tumor cell inoculation, followed by weekly recall vaccination (Figure $5 \mathrm{~A})$. Half of the mice in each group were treated with focal tumor radiotherapy given in 3 consecutive daily doses of $8 \mathrm{~Gy}$. Neo-vax did not protect mice from tumor development, but it significantly improved tumor control achieved with focal radiotherapy (Figure 5, B and C), an effect that was consistently observed in several independent experiments (Supplemental Figure 5). Spontaneous lung metastases were significantly reduced, compared with control, only in mice treated with Neo-vax and radiotherapy (Figure 5D). There was a significant increase of $\mathrm{CD} 8^{+} \mathrm{T}$ cells and a decrease of $\mathrm{CD}^{+} \mathrm{CD}^{2} 5^{+} \mathrm{FOXP}^{+}$regulatory $\mathrm{T}$ cells (Tregs) in 
A
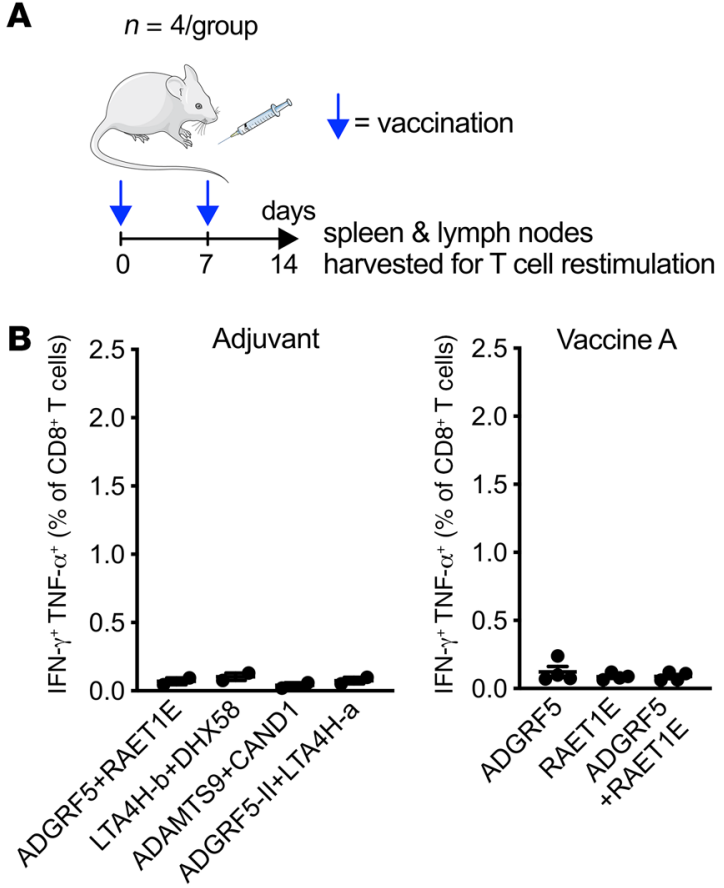

\begin{tabular}{ccc}
$\begin{array}{c}\text { Vaccination } \\
\text { groups }\end{array}$ & Neoepitopes & $\begin{array}{c}\text { MHC } \\
\text { binding }\end{array}$ \\
\hline Adjuvant & - & - \\
A & ADGRF5 / RAET1E & $\mathrm{H} 2-\mathrm{K}^{\mathrm{d}} / \mathrm{H} 2-\mathrm{L}^{\mathrm{d}}$ \\
B & LTA4H-b / DHX58 & $\mathrm{H} 2-\mathrm{K}^{\mathrm{d}} / \mathrm{H} 2-\mathrm{L}^{\mathrm{d}}$ \\
C & ADAMTS9 / CAND1 & $\mathrm{IAd} / \mathrm{H} 2-\mathrm{K}^{\mathrm{d}}$ \\
D & ADGRF5-II / LTA4H-a & $\mathrm{IA}^{\mathrm{d}} / \mathrm{H} 2-\mathrm{K}^{\mathrm{d}}$
\end{tabular}
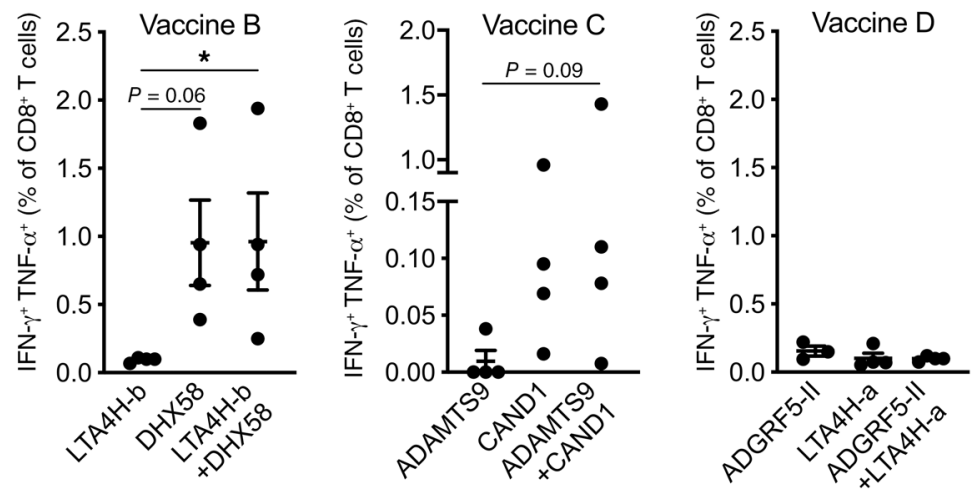

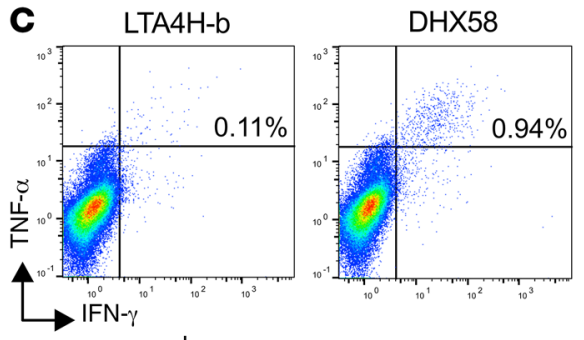

Gated on $\mathrm{CD}^{+}{ }^{+} \mathrm{T}$ cells

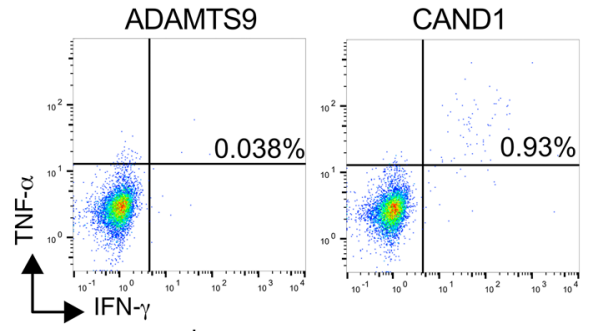

Gated on $\mathrm{CD}^{+} \mathrm{T}$ cells

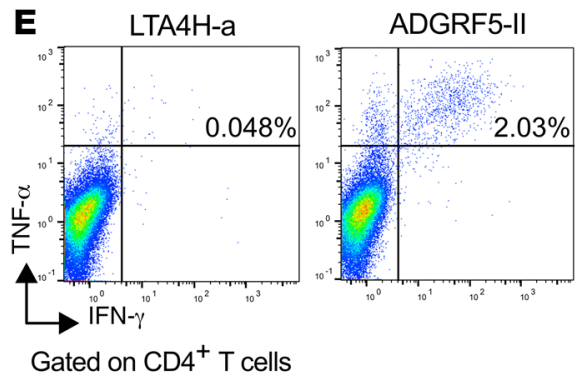

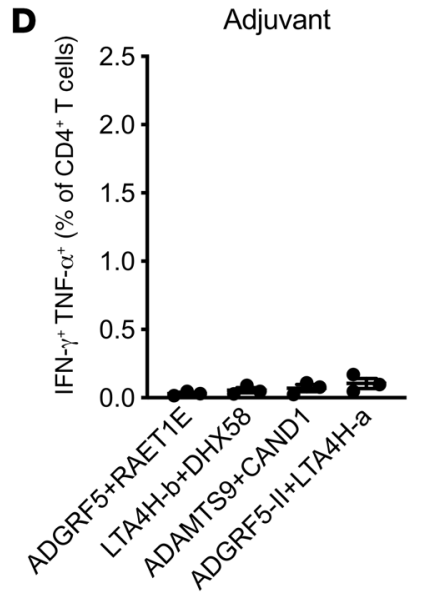
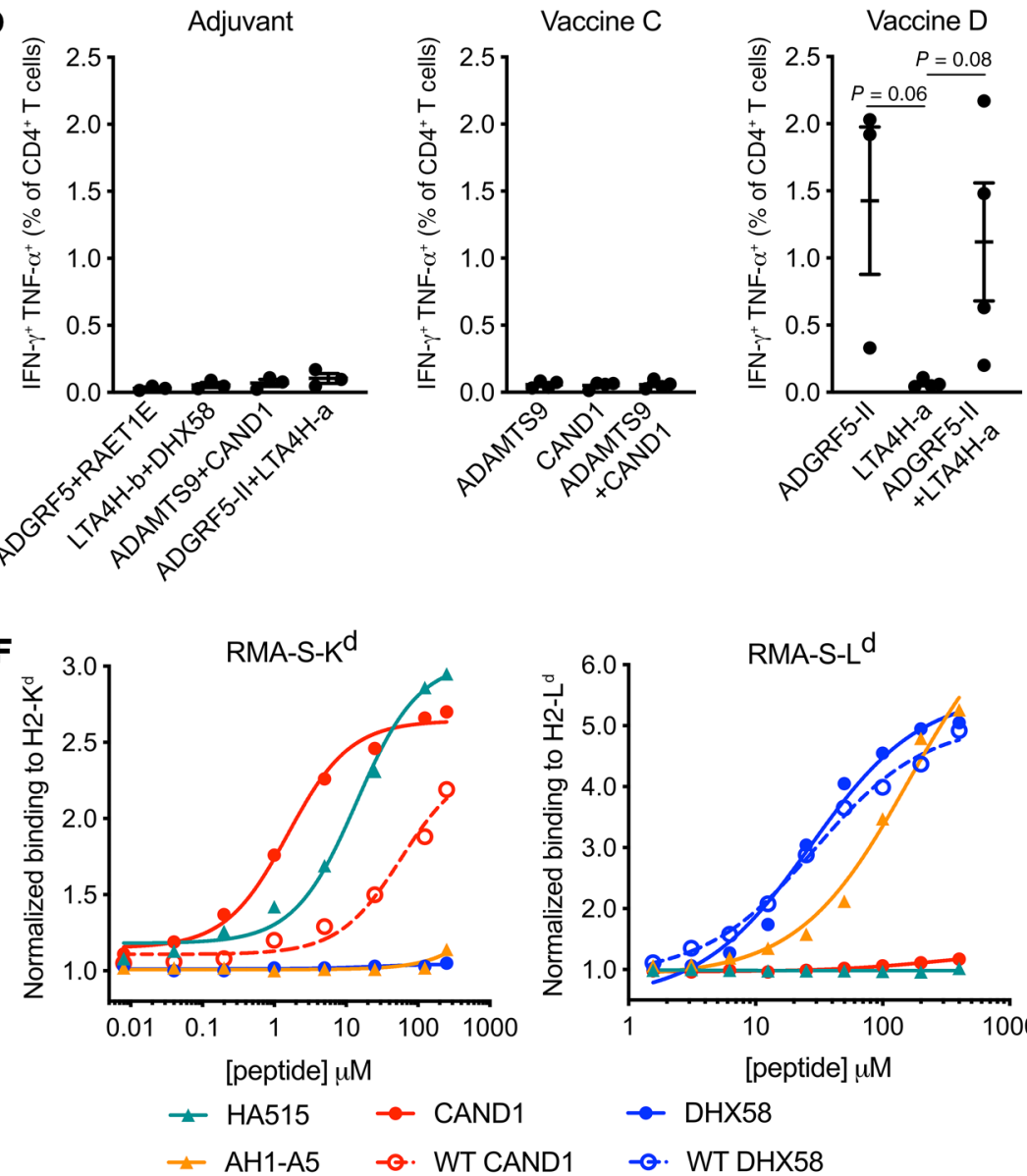

$\rightarrow$ AH1-A5 - - WT CAND1

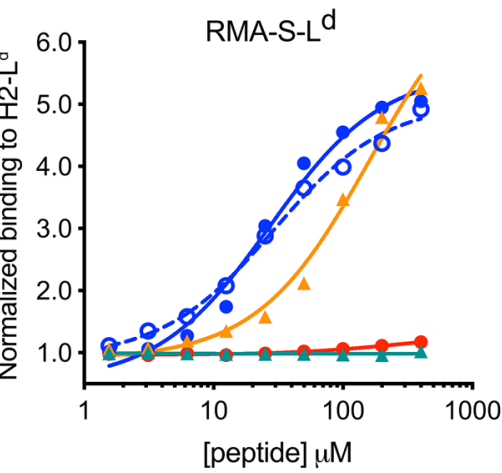

$\rightarrow$ DHX58

Q. WT DHX58 
Figure 2. Identification of immunogenic neoepitopes in 4T1 cells. (A) Experimental procedure. Mice were vaccinated twice with adjuvant alone, or with a combination of 2 neoepitopes binding to different alleles of MHC-I or MHC-II to avoid competition ( $75 \mu$ g each), as indicated ( $n=4$ per group). One week after the second vaccination, spleen and vaccine-draining lymph nodes were harvested and single-cell suspensions prepared for ex vivo stimulation experiments. (B-E) Flow cytometry analysis of IFN- $\gamma^{+}$TNF- $\alpha^{+}$ cells among $\mathrm{CD}^{+}$( $\mathbf{B}$ and $\left.\mathbf{C}\right)$ or $\mathrm{CD} 4^{+}$( $\mathbf{D}$ and $\left.\mathbf{E}\right) \mathrm{T}$ cells from mice vaccinated as indicated in $\mathbf{A}$ and stimulated ex vivo with each peptide individually or in combination. Representative flow cytometry plots of IFN- $\gamma /$ TNF- $\alpha$ intracellular staining in $\mathrm{CD}^{+}$(C) or $\mathrm{CD} 4^{+}$(E) T cells are shown after gating on viable $\mathrm{CD}^{+} \mathrm{T}$ cells. ${ }^{*} P<0.05$, with Kruskal-Wallis and Dunn's multiple-comparison tests. (F) Dose-response MHC-I binding assay comparing mutated and nonmutated (WT) DHX58 and CAND1 epitopes. RMA-S-K (left) or RMA-S- $\mathrm{L}^{\mathrm{d}}$ (right) cells were incubated with increasing concentrations of the peptides for 2 hours before determination of $\mathrm{H} 2-\mathrm{K}^{\mathrm{d}}$ or $\mathrm{H} 2-\mathrm{L}^{\mathrm{d}} \mathrm{MFI}$ by flow cytometry and normalization as in Figure $1 \mathrm{C}$.

irradiated compared with untreated tumors (Figure 5, E-G). The $\mathrm{CD}^{+} /$Treg ratio was significantly increased by Neo-vax, with a further improvement when Neo-vax was combined with radiation (Figure 5H). Consistent with the Th1 cytokine profile of ADGRF5II-specific CD $4^{+} \mathrm{T}$ cells observed in vaccinated mice (Supplemental Figure 2), the $\mathrm{T}_{-}$bet $^{+} /$Treg $\mathrm{CD} 4^{+} \mathrm{T}$ cell ratio was increased in the tumors of mice treated with Neo-vax irrespective of radiation (Figure 5I). Overall, these results suggest that neoantigen vaccination improves both local and systemic antitumor immune responses in mice treated with radiotherapy.

To further characterize the neoantigen-specific T cell response, we stained tumor-infiltrating T cells with $\mathrm{H} 2-\mathrm{K}^{\mathrm{d}}$ dextramers loaded with the CAND1 neoepitope. We observed a significant increase in CAND1-specific CD8 ${ }^{+} \mathrm{T}$ cells in the tumors of mice treated with radiation plus Neo-vax in comparison with control mice (Figure 6, $\mathrm{A}-\mathrm{C}$ ). The frequency and counts of CAND1-specific T cells were also increased in tumors of mice treated with either Neo-vax or radiation, albeit to a lower extent than when the 2 treatments were combined, suggesting a contribution of radiation to priming of CAND1-specific CD ${ }^{+} \mathrm{T}$ cells. In the tumor-draining lymph nodes, IFN- $\gamma$ production after ex vivo stimulation with the $2 \mathrm{CD} 8^{+}$ neoepitopes (DHX58 and CAND1) was seen only when Neo-vax was combined with radiotherapy (Figure 6, D and E), further supporting a role for radiation in enhancing $\mathrm{T}$ cell responses directed against radiation-upregulated neoantigens. In contrast, IFN- $\gamma$ production after stimulation with the $\mathrm{CD}^{+}$neoepitope ADGRF5-II was driven by Neo-vax, regardless of radiotherapy (Figure 6F).

Therapeutic vaccination with radiation-upregulated neoantigens improves tumor control and survival. The above experiments demonstrated that the antitumor $\mathrm{T}$ cells activated by Neo-vax were able to inhibit the growth of irradiated $4 \mathrm{~T} 1$ tumor and its lung metastases. However, in these experiments (Figure 5, A-D, and Supplemental Figure 5) vaccination was started before tumor inoculation. To test whether therapeutic vaccination was effective, mice were immunized after tumor inoculation, followed a few days later by focal tumor radiotherapy (Figure 7A). We observed a significant delay in tumor progression in mice treated with radiotherapy plus Neo-vax in comparison with radiotherapy alone, although the effect was delayed (Figure 7, B and C) in comparison with the effect observed with prophylactic vaccination (Figure $5 \mathrm{~B}$ and Supplemental Figure 5), suggesting that neoantigen-specific $\mathrm{T}$ cells expansion may occur slowly and require at least 1 vaccination boost. Nevertheless, vaccination resulted in a small but significant improvement in survival (Figure 7D).

Neoantigen-specific $C D 8^{+}$and $C D 4^{+} T$ cells are required for the efficacy of the neoantigen vaccine. It has recently been demonstrated that success of the antitumor immune response depends on the expression by the tumor of neoantigens that activate both $\mathrm{CD} 4^{+}$ and $\mathrm{CD} 8^{+} \mathrm{T}$ cells (29). To test the contribution of the MHC-II neoepitope ADGRF5-II to the antitumor immune response, mice were vaccinated with the 3 peptides, or only with DHX58 and CAND1. In the absence of ADGRF5-II, the ability of vaccination to improve control of the irradiated $4 \mathrm{~T} 1$ tumors was abrogated (Figure $8 \mathrm{~A}$ ). To understand the bases for this loss of activity, tumor-draining lymph nodes were harvested on day 31 and tested for IFN- $\gamma$ production in response to stimulation with the neoantigens ex vivo. Inclusion of the MHC-II neoantigen in the vaccine was not required for priming of CAND1 and DHX58-specific CD8 ${ }^{+}$T cells (Figure 8B). ADGRF5II-specific $\mathrm{CD} 4^{+} \mathrm{T}$ cells produced large amounts of IFN- $\gamma$ after peptide restimulation. Moreover, the induction of IFN- $\gamma$ by the $\mathrm{CD}^{+}$ epitope AH1-A5, although lower in magnitude compared with neoantigen-specific $\mathrm{T}$ cells, suggested that $\mathrm{CD} 4^{+} \mathrm{T}$ cell activation by the vaccine was required to promote antigenic spread (Figure 8B). This interpretation is consistent with recent data testing the effects of vaccination with $\mathrm{CD} 4^{+} \mathrm{T}$ cell neoepitopes in combination with focal tumor radiotherapy in the CT26 tumor model (30). To evaluate the cytotoxic activity of neoantigen-specific $\mathrm{T}$ cells, splenocytes were harvested from 4T1-bearing mice vaccinated with DHX58 and CAND1 or with Neo-vax. After in vitro restimulation with CAND1 or ADGRF5-II, $\mathrm{CD}^{+}$and $\mathrm{CD} 4^{+} \mathrm{T}$ cells were isolated and used as effector cells for in vitro killing assays with $4 \mathrm{~T} 1$ as target cells. Relative killing of irradiated $4 \mathrm{~T} 1$ cells was significantly higher when $\mathrm{CD}^{+} \mathrm{T}$ cells were obtained from mice that received the Neo-vax, suggesting that the presence of helper $\mathrm{CD} 4^{+} \mathrm{T}$ cells improved systemic expansion of tumor-specific cytotoxic $\mathrm{CD}^{+} \mathrm{T}$ cells (Figure $8 C)$. Unexpectedly, there was also a significant killing of irradiated $4 \mathrm{~T} 1$ cells by $\mathrm{CD} 4^{+} \mathrm{T}$ cells (Figure $8 \mathrm{C}$ ). In addition, depletion of either $\mathrm{CD}^{+}$or $\mathrm{CD} 4^{+} \mathrm{T}$ cells abrogated the effect of Neo-vax (Figure 8D), demonstrating the requirement for both $\mathrm{T}$ cell populations.

To confirm the ability of neoantigen-specific $\mathrm{CD} 4^{+} \mathrm{T}$ cells to kill $4 \mathrm{~T} 1$ cells, tumor-draining lymph node cells were harvested from mice vaccinated with adjuvant control or with CAND1 and ADGRF5-II neoantigens and treated as above for ex vivo killing assays. Killing of irradiated 4T1 cells was decreased by blockade of MHC-I and MHC-II (Figure 9, A-C, and Supplemental Figure 6), indicating the contribution of both $\mathrm{CD}^{+}$and $\mathrm{CD} 8^{+} \mathrm{T}$ cells. Cytotoxicity by $\mathrm{CD} 4^{+} \mathrm{T}$ cells has been shown to be dependent on FAS expression by target cells (31). Consistently, expression of Fas by 4T1 cells was significantly enhanced by radiation (Figure 9D), and blockade of FAS ligand (FASL) reduced killing of irradiated 4T1 cells by lymph node T cells from neoantigen-vaccinated mice (Figure 9C). Radiation also upregulated the expression of TnfrsflOb (encoding the death receptor DR5) in 4T1 cells, and a small but significant reduction in irradiated $4 \mathrm{~T} 1$ cell killing was seen upon blockade of the DR5 ligand TRAIL (Figure 9, C and D).

Overall, these results indicate that targeting of $\mathrm{CD} 8^{+}$and $\mathrm{CD} 4^{+}$ neoantigens is required for optimal antitumor activity by neoepi- 


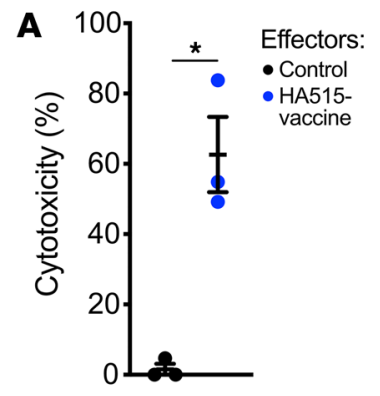

Targets: HA515
B

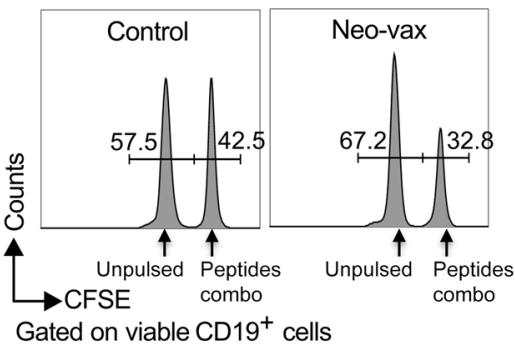

C

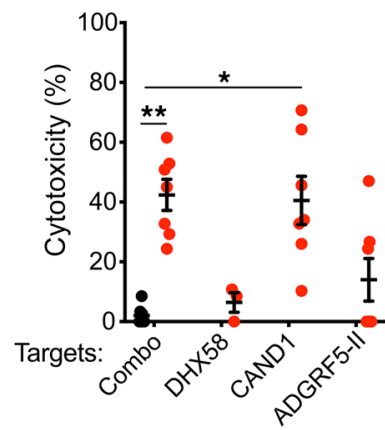

Spleen

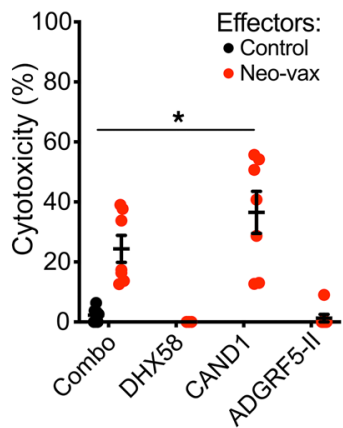

D

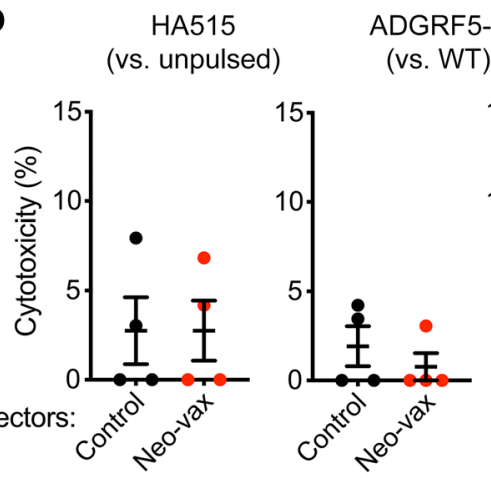

CAND1 (vs. WT)
CAND1

(vs. WT)

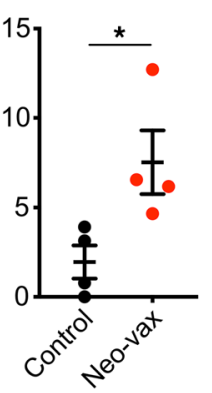

+ MHC-I

E

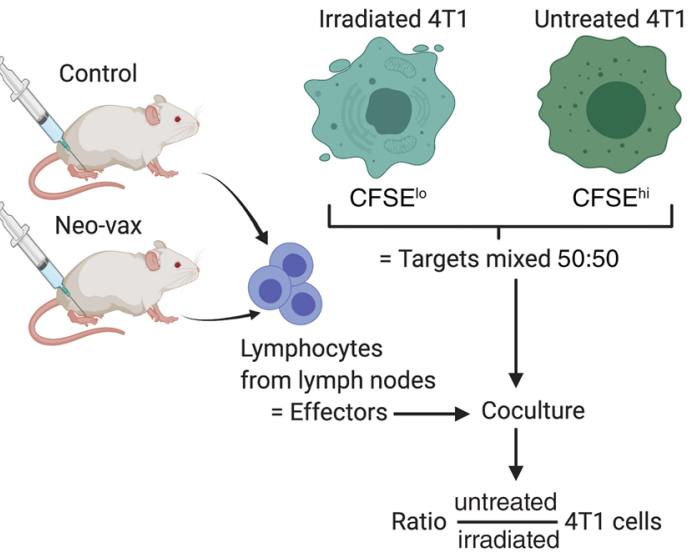

blockade

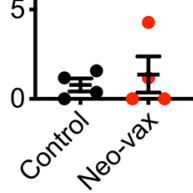

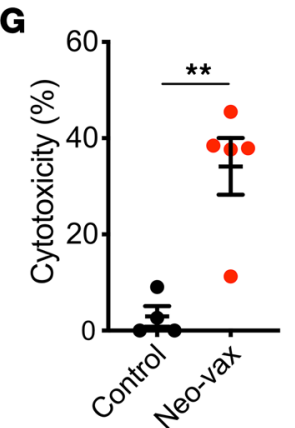

Before coculture | After coculture with effectors:
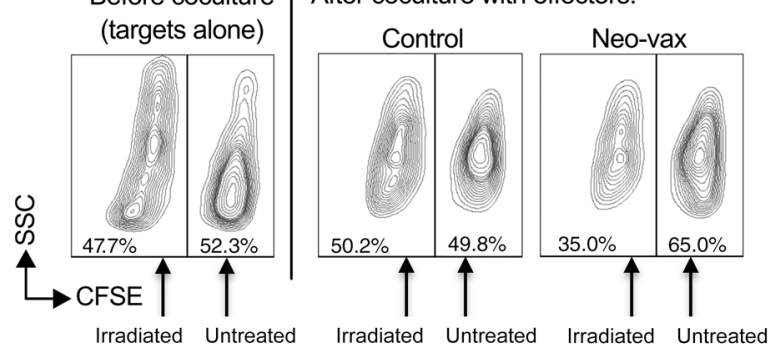

$\mathbf{F}$

Figure 3. CAND1-specific CD8 ${ }^{+} \mathbf{T}$ cells are cytotoxic and kill preferentially irradiated 4 T1 cells. Mice were vaccinated twice with adjuvant alone (Control), with HA515, or with the 3 immunogenic neoepitopes (DHX58, CAND1, and ADGRF5-II; Neo-vax). (A-C) One week after the last vaccination, mice were injected i.v. with CFSE-labeled target cells: CFSE ${ }^{\text {hi }}$ cells were pulsed with indicated peptides, whereas CFSE ${ }^{l o}$ cells were unpulsed. Lymph nodes and spleen were harvested 24 hours later, and pulsed and unpulsed target cells were quantified by flow cytometry after gating on viable CD19+ CFSE ${ }^{+}$cells. (A) Percentage of cytotoxicity toward HA515-paulsed target cells ( $n=3$ mice per group), calculated as indicated in Methods. ${ }^{*} P<0.05$, with unpaired 2-tailed Welch's $t$ test. (B) Representative flow cytometry plots showing the killing of target cells in lymph nodes. (C) Percentage of cytotoxicity toward neoepitope-pulsed target cells determined in the lymph nodes and spleen ( $n=3-7$ mice per group). ${ }^{*} P<0.05$, ${ }^{* *} P<0.01$, with Kruskal-Wallis and Dunn's multiple-comparison tests. Data are pooled from 2 independent experiments. (D) Vaccine-draining lymph node cells were co-cultured with CFSE target cells pulsed with indicated neoepitopes, whereas CFSE $^{10}$ cells were pulsed with WT peptides. For HA515 peptide, CFSE ${ }^{10}$ cells were unpulsed. MHC-I-blocking antibody was added as indicated. After incubation for 16-18 hours, cells were harvested for flow cytometry quantification of viable CD19+CFSE ${ }^{+}$target cells, and the percentage of cytotoxicity toward mutated versus WT peptide-loaded targets was calculated. ${ }^{*} P<0.05$, with unpaired 2 -tailed Welch's $t$ test. Data are representative of 2 independent experiments. (E-G) In vitro killing of $4 T 1$ target cells was tested as described in the schema (E). (F) Representative flow plots after gating on viable CFSE+ cells. (C) Percentage of cytotoxicity toward irradiated cells versus untreated cells, calculated as described in Methods. ${ }^{* *} P<0.01$, with unpaired 2-tailed Welch's $t$ test. All data are expressed as mean \pm SEM. tope vaccines. In the context of radiation therapy, neoantigenspecific $\mathrm{CD} 4^{+} \mathrm{T}$ cells may contribute to the therapeutic efficacy of the vaccine not only by fostering the effector function of $\mathrm{CD}^{+} \mathrm{T}$ cells, but also by directly killing the cancer cells, reflecting the ability of radiation to upregulate the expression of MHC-II antigens and death receptors on the cancer cells.

\section{Discussion}

Here we describe a fundamental role of radiotherapy in exposing immunogenic mutations to the immune system. The tumor mutational burden correlates with responses to ICB across different tumor types (1-4), but significant overlap is present between responders and nonresponders, suggesting that the expression lev- 
A
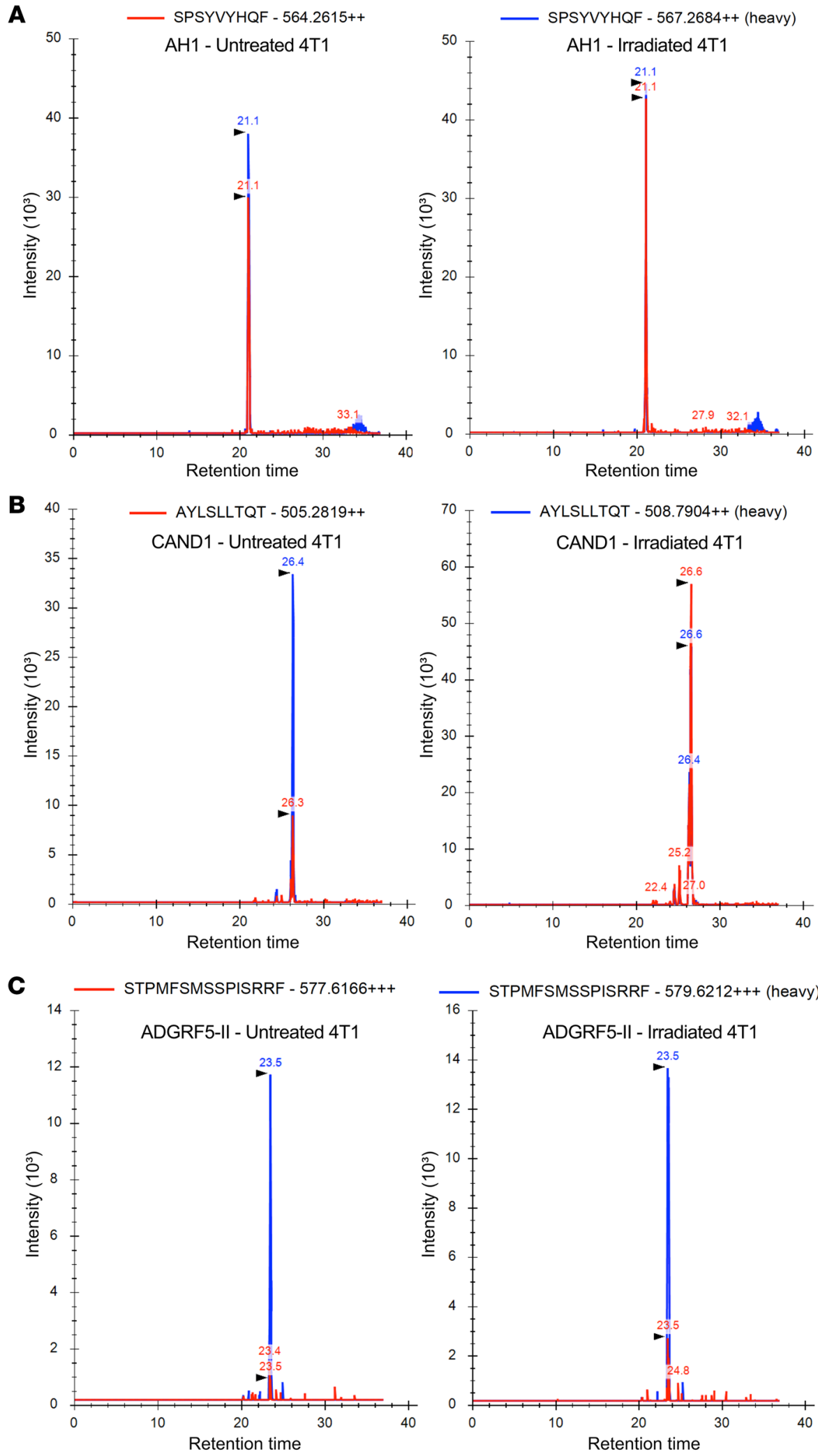

Figure 4. Targeted detection and quantification of neoepitopes and control epitope AH1 at the surface of tumor cells after irradiation. SRM chromatograms of endogenous peptides (in red) and isotope-labeled peptides (in blue) for the AH1 epitope (SPSYVYHQF) (A), the CAND1 neoepitope (AYLSLLTQT) (B), and the ADCRF5-II neoepitope (STPMFSMSSPISRRF) (C). Detection and quantification of the peptides from untreated (left panels) or irradiated (right panels) 4T1 cells were performed by SRM analysis as described in Methods. Additionally, the MS/MS spectra for CAND1 and ADCRF5-II are shown in Supplemental Figures 3 and 4. els of an antigenic mutation, and not only its presence in the genome, are critical $(32,33)$. This notion is consistent with the well-established importance of the antigen rate of synthesis and degradation and overall abundance for presentation in the MHC-I pathway $(13,14)$. Our data highlight the concept that expression of antigenic mutations targeted by antitumor $\mathrm{T}$ cells in cancer cells can be modulated by treatment. Among the genes encoding mutations that were confirmed to generate neoepitopes able to bind to MHC-I, we identified one gene, Lta4h, that was decreased in expression following radiation. Although the neoepitopes encoded by $L t a 4 h$ were not immunogenic in vivo, these results nevertheless raise the possibility that in some cases radiation could reduce neoantigen presentation.

Inspired by the identification of $\mathrm{CD} 8^{+} \mathrm{T}$ cells specific for an immunogenic mutation upregulated by radiotherapy in a patient with NSCLC who experienced a complete response to radiotherapy and blockade of the inhibitory receptor CTLA-4 (19), we selected 4T1 mouse carcinoma cells, which possess a number of mutations similar to those found in our patient's tumor, to investigate the ability of radiotherapy to expose immunogenic mutations. Using a rigorous pipeline to select genes that were expressed in vitro and in vivo, and significantly upregulated by radiotherapy, we identified 4 genes of interest. Upregulation of Raet1e and Dhx58 expression is known to occur in irradiated cells $(34,35)$. Cand 1 encodes a regulator of cullin-RING ubiquitin ligases involved in ubiquitinylation of proteins degraded by the proteasome. Given the increased protein degradation observed after radiation (17), the upregulation of Cand1 may occur as part of this process. Finally, Adgrf5 is an adhesion G proteincoupled receptor implicated in pulmonary surfactant homeostasis that has not been previously studied in the context of radiation (36). Regardless of the possible function of these proteins in the cellular response to radiation, each gene was found to encode a mutated peptide capable of binding to MHC-I, but only CAND1 and DHX58 elicited CD8 ${ }^{+} \mathrm{T}$ cell responses in vivo. As previously reported for immunogenic mutations that were selected using MHC-I binding algorithms 
Table 2. Quantification of (neo)epitopes in untreated and irradiated 4T1 cells

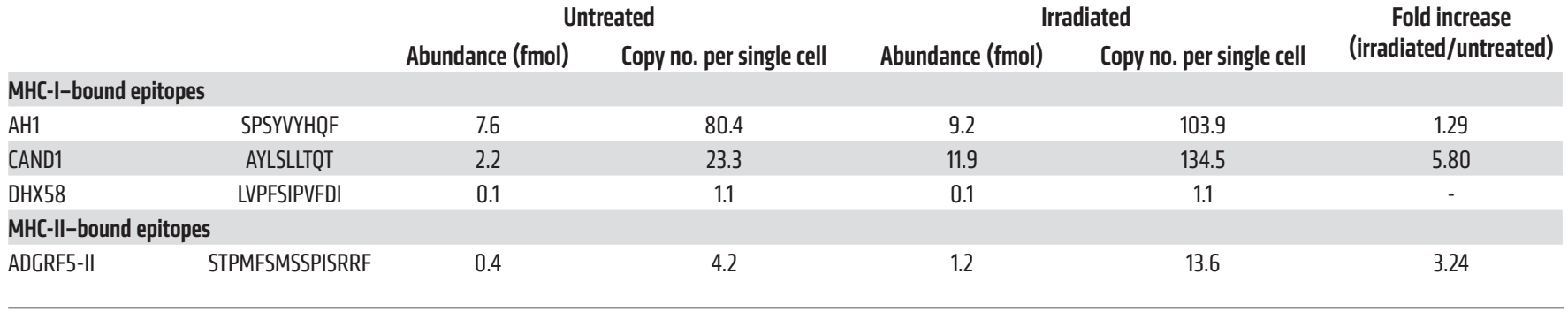

but were found to elicit $\mathrm{CD} 4^{+} \mathrm{T}$ cell responses (5), the mutation in the Adgrf5 gene did not elicit $\mathrm{CD} 8^{+} \mathrm{T}$ cell responses but was effective at inducing $\mathrm{CD} 4^{+} \mathrm{T}$ cell responses. The $\mathrm{CD} 4^{+}$neoantigenspecific response was required for the therapeutic efficacy of the combination of radiotherapy and neoantigen vaccine, consistent with previous reports highlighting the importance of immunogenic mutations in MHC-II epitopes $(25,29,37,38)$.

Among the $2 \mathrm{CD}^{+} \mathrm{T}$ cell neoepitopes encoded by genes upregulated by radiation, we identified CAND1 in the immunopeptidome eluted from MHC-I of 4T1 cells and demonstrated that it was increased almost 6-fold in irradiated cells. In contrast, we were unable to confirm the presentation of DHX58 by $4 \mathrm{~T} 1$ cells, despite the fact that this neoepitope induced IFN- $\gamma$ responses in lymph nodes draining irradiated $4 \mathrm{~T} 1$ tumors. It is likely that DHX58 is presented in small amounts by $4 \mathrm{~T} 1$ cells, below the detection limit of the assay we used. Somewhat unexpectedly, we found that untreated $4 \mathrm{~T} 1$ cells presented small quantities of ADGRF5-II despite the minimal expression of MHC-II, with an over 3-fold increase after radiation. The induction of MHC-II expression on $4 \mathrm{~T} 1$ cells by radiation and IFN- $\gamma$ suggests that $\mathrm{CD} 4^{+}$ $\mathrm{T}$ cells can directly interact with the cancer cells during radiation-induced tumor rejection.

While the antitumor immune response against neoantigens expressed by solid tumors is predominantly attributed to $\mathrm{CD} 8^{+} \mathrm{T}$ cells, CD $4^{+} \mathrm{T}$ cells are also important drivers of antitumor immunity $(25,38,39)$. Most solid tumors do not express MHC-II molecules, but the antigen-presenting cells present in the tumor microenvironment capture dead tumor cells and present tumor antigens to $\mathrm{CD}^{+} \mathrm{T}$ cells. A recent report by Alspach and colleagues demonstrated the need for expression of both MHC-I and MHC-II neoantigens by an MHC-II-negative tumor at the site of tumor regression, indicating a role for $\mathrm{CD}^{+} \mathrm{T}$ cells not only during priming but also at the effector phase of the antitumor immune response (29). Furthermore, a direct cytotoxic activity of $\mathrm{CD}^{+}{ }^{+} \mathrm{T}$ cells toward tumor cells in an MHC-II-dependent manner was recently demonstrated in human bladder cancer (40). Radiation-induced cell death promotes transfer of tumor antigens to myeloid cells in the tumor microenvironment (41), which could explain the synergy we observed between vaccination with MHC-I and MHC-II neoantigens and tumor irradiation. In addition, radiation-induced expression of MHC-II on the $4 \mathrm{~T} 1$ cancer cells, together with the upregulation of FAS, resulted in direct cytotoxic activity of the ADGRF5-II-specific CD4 ${ }^{+} \mathrm{T}$ cells against irradiated $4 \mathrm{~T} 1$ cells. Cytotoxicity by $\mathrm{CD} 4^{+} \mathrm{T}$ cells usually requires FAS expression by target cells (31), but antibody blocking experiments suggest a small contribution for DR5 as well. Since the direct cytotoxic activity of $\mathrm{CD} 4^{+} \mathrm{T}$ cells against irradiated $4 \mathrm{~T} 1$ cells was demonstrated in vitro, however, we cannot exclude the possibility that tumor control in vivo requires indirect antigen presentation by other cell types, such as tumor-infiltrating macrophages (42).

Vaccination of mice with CAND1, DHX58, and ADGRF5-II improved the control of irradiated 4T1 tumor and nonirradiated lung metastases, suggesting that it promoted antigenic spread. However, no cures were achieved, a result that is consistent with other studies testing vaccination strategies against $4 \mathrm{~T} 1$ carcinoma $(43,44)$. Thus, additional interventions will be required to overcome the immunosuppressive environment generated by this aggressive carcinoma and achieve cures.

In summary, we provided proof-of-principle evidence that radiotherapy not only boosts the expression of genes encoding immunogenic neoepitopes that are presented by MHC-I and MHC-II molecules and elicit $\mathrm{CD}^{+}$and $\mathrm{CD} 4^{+} \mathrm{T}$ cell responses, but also favors MHC-II and death receptor upregulation. Thus, radiotherapy stands out as an ideal combinatorial partner to improve the efficacy of personalized neoantigen vaccination approaches currently pursued in the clinic $(9,10,45)$.

\section{Methods}

Cell culture. 4T1 cells were obtained from F. Miller (then at Michigan Cancer Foundation, Detroit, Michigan), who originally isolated this cell line (46), and a large stock of low-passage frozen cells was prepared. Cells were authenticated by IDEXX Bioresearch in 2019 and routinely screened for Mycoplasma (LookOut Mycoplasma PCR Detection kit, Sigma-Aldrich). The transporter associated with antigen processing-deficient (TAP-deficient) RMA-S cells are derived from a C57BL/6 T cell lymphoma (47). RMA-S cells transfected with H2- $L^{d}$ (RMA-S-L ${ }^{\text {d }}$ or with $H 2-K^{d}$ (RMA-S-K $\mathrm{K}^{\mathrm{d}}$ cDNA were provided by Ted H. Hansen (Washington University School of Medicine, St. Louis, Missouri, USA; ref. 48). Cells were cultured in DMEM (Invitrogen) supplemented with $2 \mathrm{mmol} / \mathrm{L}$ L-glutamine, $100 \mathrm{U} / \mathrm{mL}$ penicillin, 100 $\mu \mathrm{g} / \mathrm{mL}$ streptomycin, $2.5 \times 10^{-5} \mathrm{~mol} / \mathrm{L} 2$-mercapthoethanol, and $10 \%$ FBS (Life Technologies). For RMA-S-K ${ }^{\mathrm{d}}$ and RMA-S-L ${ }^{\mathrm{d}}$ cells, G418 (InvivoGen) was added at 400 and $600 \mu \mathrm{g} / \mathrm{mL}$, respectively. For primary cell culture, complete T cell medium was used: RPMI (Invitrogen) supplemented with $2 \mathrm{mmol} / \mathrm{L}$ L-glutamine, $100 \mathrm{U} / \mathrm{mL}$ penicillin, $100 \mu \mathrm{g} / \mathrm{mL}$ streptomycin, $2.5 \times 10^{-5} \mathrm{~mol} / \mathrm{L}$ 2-mercapthoethanol, $10 \%$ FBS, and recombinant human IL-2 (30 U/mL) (Biological Resources Branch, National Cancer Institute, Bethesda, Maryland). 

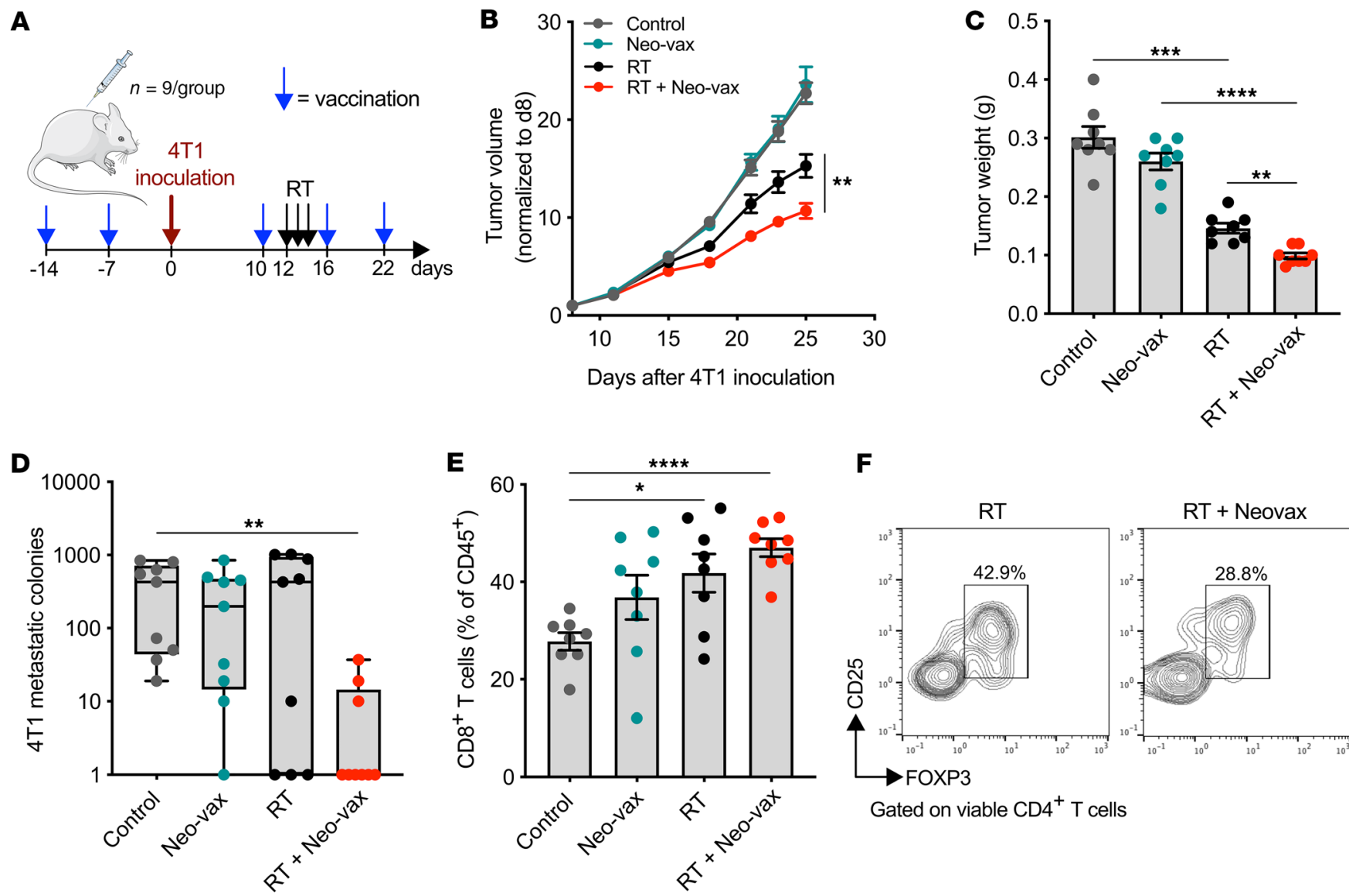

$\mathbf{F}$
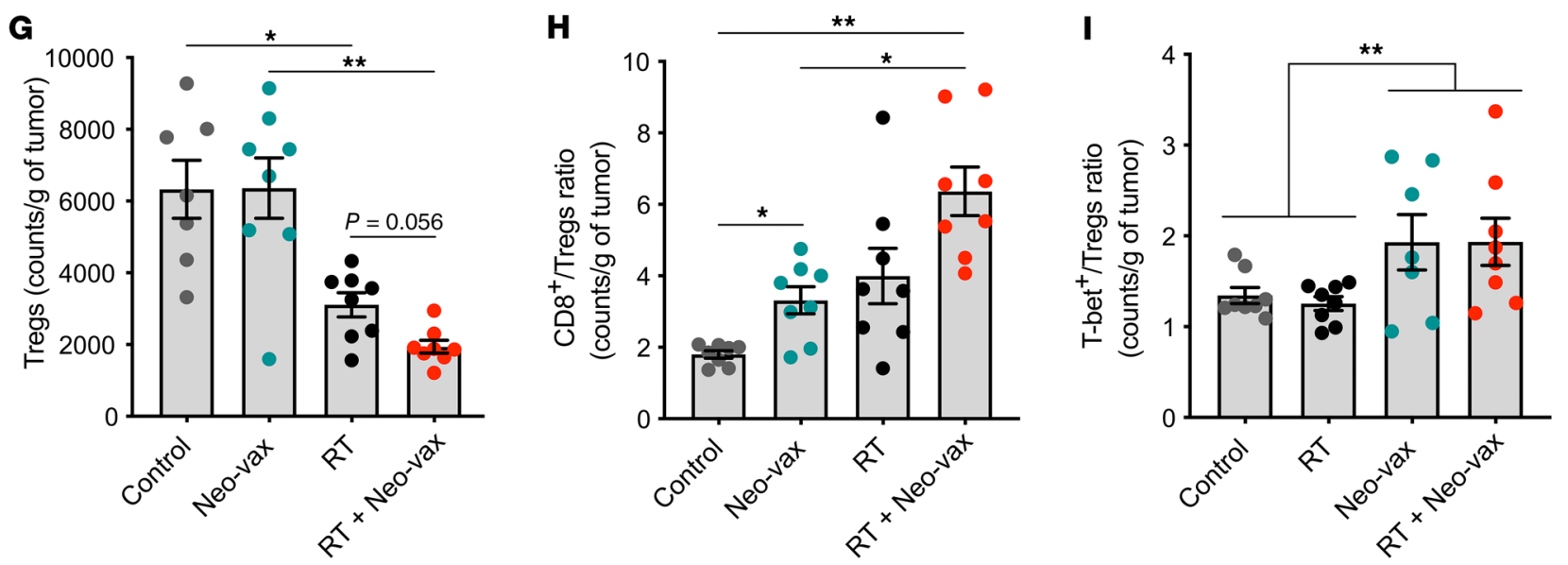

Figure 5. Vaccination with radiation-upregulated neoantigens enhances the antitumor immune response in combination with radiotherapy. (A) Experimental schema. Mice were vaccinated twice with adjuvant alone (Control) or with the combination of 3 immunogenic neoepitopes (DHX58, CAND1, and ADGRF5-II; Neo-vax). One week after the second vaccination, 4T1 cells were injected s.c. in the right flank of mice. Vaccination was continued once a week. Tumors were irradiated with a dose of 8 Gy each on 3 consecutive days (RT). (B) Tumor volume measured over time after 4T1 cell inoculation ( $n=9$ per group). Statistical significance of Neo-vax + RT was assessed by repeated-measures 2-way ANOVA. (C) Tumor weight at day 25 ( $n=8)$. (D) Number of clonogenic 4T1 lung metastases at day 25. Statistical significance was assessed by Kruskal-Wallis and Dunn's multiple-comparison tests $(n=9)$. (E-I) Flow cytometry analysis of the tumors at day $25(n=8)$. (E) Percentages of CD8 ${ }^{+}$T cells among viable CD45+ cells. (F) Representative flow plots of regulatory $T$ cells (Tregs; $\mathrm{CD25}{ }^{+} \mathrm{FOXP3}^{+}$) after gating on $\mathrm{CD} 4^{+} \mathrm{T}$ cells. (G) Absolute counts (normalized to tumor weight) of intratumoral Tregs and ratio of CD8 $8^{+}$cells to Tregs (H) or T-bet ${ }^{+}$cells to Tregs (I). All data presented here are expressed as mean \pm SEM. Comparisons between groups were made with Welch's ANOVA and Dunnett's T3 multiple-comparison tests, except in I, where the effect of Neo-vax was assessed by ordinary 2 -way ANOVA. In all panels, ${ }^{*} P<0.05$, ${ }^{* *} P<0.01,{ }^{* *} P<0.001,{ }^{* * * *} P<0.0001$. 
A

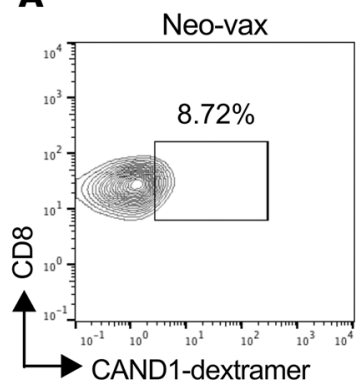

Gated on viable $\mathrm{CD} 8^{+}{ }^{\top}$ cells

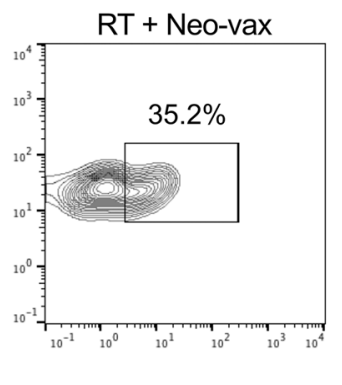

B

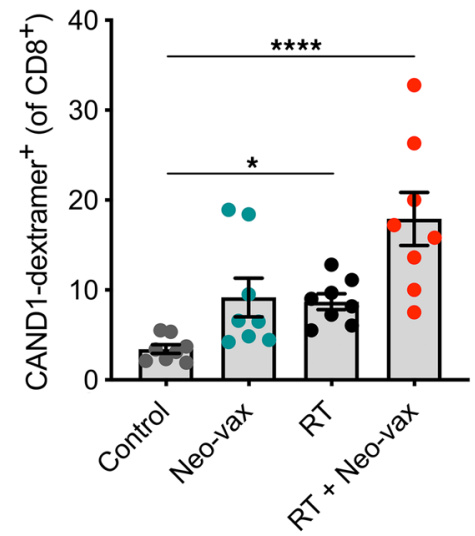

C

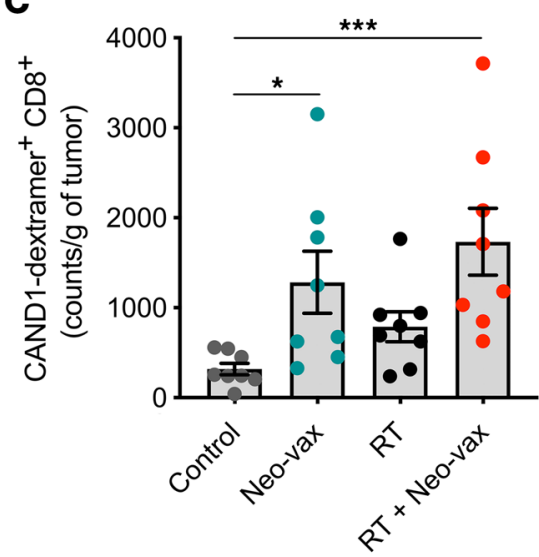

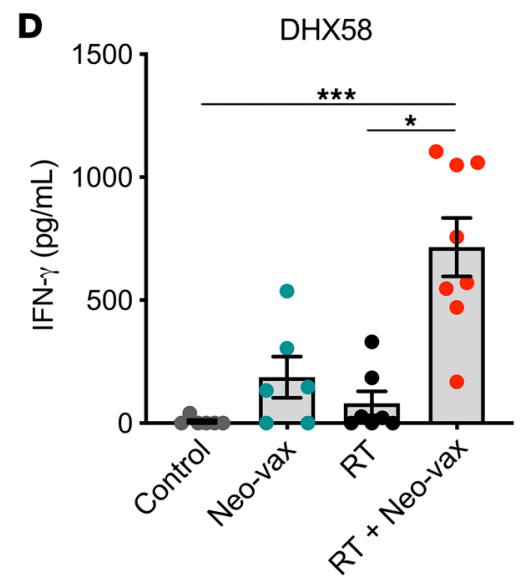

E

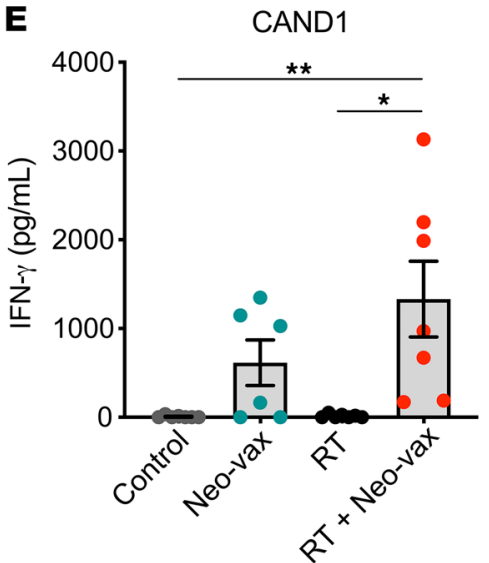

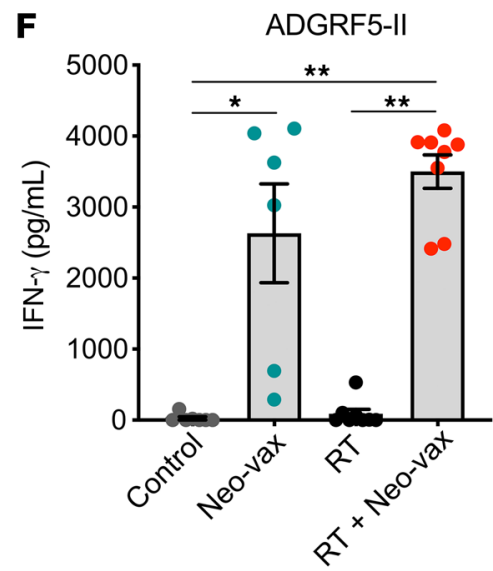

Figure 6. Radiotherapy enhances $T$ cell responses against radiation-upregulated neoantigens. Mice were treated as in Figure $5 \mathrm{~A}$, and tumors and draining lymph nodes were harvested on day 25 for analysis. (A-C) Representative flow plots (A), percentages (B), and absolute counts (C) of CAND1dextramer-positive cells among intratumoral $\mathrm{CD} 8^{+} \mathrm{T}$ cells. Data are presented as mean $\pm \mathrm{SEM}$. Comparisons between groups were made with KruskalWallis and Dunn's multiple-comparison tests $(n=8)$; ${ }^{*} P<0.05$, ${ }^{* * *} P<0.001$, ${ }^{* * *} P<0.0001$. (D-F) Secreted IFN- $\gamma$ in the supernatant of tumor-draining lymph node cells 48 hours after in vitro stimulation with DHX58 (D), CAND1 (E), and ADGRF5-II (F). Comparisons between all groups were made with Kruskal-Wallis and Dunn's multiple-comparison tests; ${ }^{*} P<0.05,{ }^{* *} P<0.01,{ }^{* * *} P<0.001(n=6-8)$.

Reagents. Peptides were synthesized by GenScript with a purity $\geq 85 \%$. Peptides were dissolved in distilled water or in DMSO and then further diluted with water, based on the manufacturer's solubility report. $\mathrm{CpG}$ oligodinucleotide (ODN 1826) and high-molecular weight poly(I:C) were purchased from InvivoGen. CD8a (clone 2.43, used for $\mathrm{CD}^{+} \mathrm{T}$ cell depletion), CD4 (clone GK1.5, used for CD4 ${ }^{+} \mathrm{T}$ cell depletion), rat IgG2b isotype control (clone LTF-2), MHC class I (H2) clone M1/42.3.9.8 (catalog BE0077), MHC class II (IA/IE) clone M5/114 (catalog BE0108), and FASL clone MFL3 (catalog BE0319) antibodies were purchased from Bio X Cell. TRAIL clone N2B2 antibody was purchased from BioLegend. The allophycocyanin-labeled (APC-labeled) MHC class I (H2-K $\mathrm{K}^{\mathrm{d}}$ ) dextramer loaded with CAND1 (AYLSLLTQT) was purchased from Immudex.

Neoantigen identification. Total genomic DNA (gDNA) and total RNA from 4T1 cells cultured in vitro were extracted using the GeneJET gDNA purification kit (Thermo Fisher Scientific) and the RNeasy Plus Mini Kit (Qiagen), respectively, according to the manufacturers' instructions. As a control, we used gDNA isolated from the tail of naive BALB/c mice. For whole-exome sequencing (WES), DNA libraries were prepared from isolated gDNA using the Kapa
Hyper DNA library preparation kit (Kapa Biosystems). Briefly, DNA samples were fragmented to an average size range of $200 \mathrm{bp}$ using a Covaris E220 shearing instrument. Adapter-ligated libraries were generated and enriched by PCR. Then the pooled libraries were hybridized with whole-exome SeqCap EZ probe pool. Finally, samples were run on HiSeq4000 sequencer (Illumina) with pairedend reads of $2 \times 100$ cycles. The sequencing reads were cleaned by trimming of adapter sequences and low-quality bases using cutadapt v1.9.1 (49), and were aligned to the mouse reference genome (GRCm38) using Burrows-Wheeler Aligner (BWA) v0.7.8 (50). The aligned BAM file was further refined by duplicate removal with Picard toolkit v1.110 (http://broadinstitute.github.io/picard). Indel realignment and base quality recalibration were performed with the Genome Analysis Toolkit v2.8-1 (51). Somatic variants were detected using VarScan2 v2.3.7 (52). For RNA sequencing (RNA-Seq), libraries were prepared using the TruSeq Stranded mRNA Sample Library Preparation kit (Illumina), according to the manufacturer's instructions. The normalized cDNA libraries were pooled and sequenced on a HiSeq4000 sequencer (Illumina) with paired-end reads of 75 cycles. The sequencing reads were cleaned as described 
A

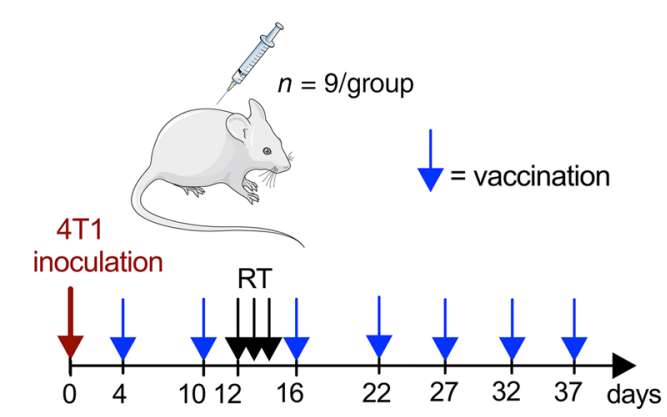

C

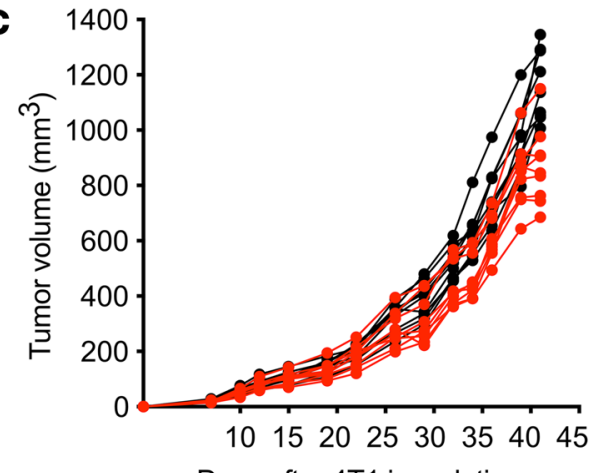

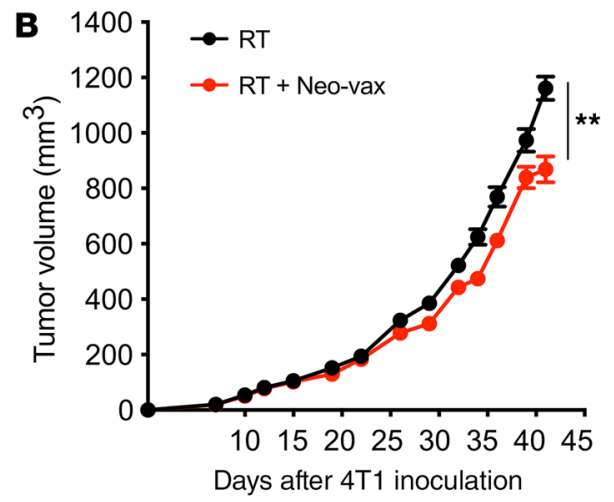

D

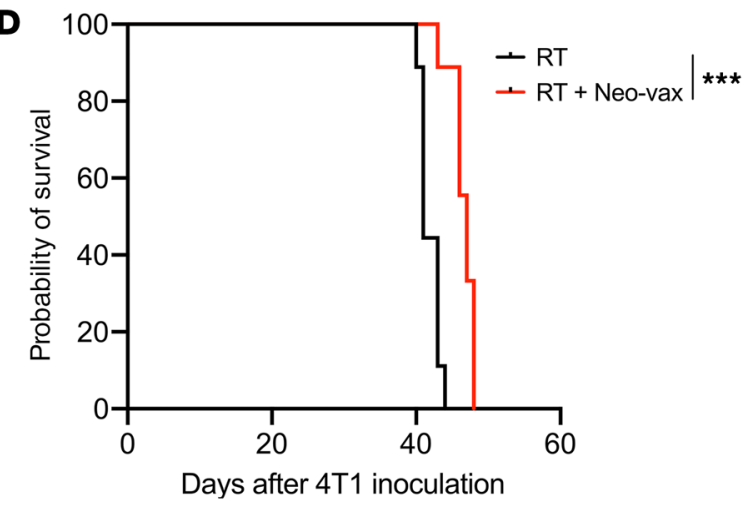

Figure 7. Therapeutic efficacy of the neoantigen vaccine in combination with radiotherapy. (A) Experimental schema. Vaccination was started on day 4 after tumor inoculation and continued every 5-6 days thereafter. Tumors were irradiated with a dose of 8 Gy on 3 consecutive days (RT). (B and C) Tumor volume over time after $4 \mathrm{~T} 1$ cell inoculation ( $n=9$ per group), shown as mean $\pm \mathrm{SEM}$ (B) and as individual mouse curves (C). Statistical significance of RT + Neo-vax was assessed by repeated-measures 2-way ANOVA; ${ }^{* *} P<0.01$. (D) Mouse survival. ${ }^{* *} P<0.001$, with log-rank test.

for the WES and were aligned to the mouse reference genome using STAR v2.5.2b (53). Raw read counts per gene were extracted using HTSeq-count v0.6.1 (54). Differential expression analysis was performed using DESeq2 v1.6.3 (55). Before prediction of neoantigens, variant call format files containing somatic mutations were subjected to standard variant effect predictor, downstream, and wild-type annotations, and filtered for frameshift and missense mutations. Additionally, variant allele frequency (VAF) data were generated using bam-readcount v0.8.0. Variants were filtered on normal tissue VAF $(<2 \%)$, tumor DNA and RNA VAF $(>5 \%)$, and tumor RNA variant coverage ( $>10$ reads). WES and RNA-Seq analyses were also performed on $4 \mathrm{~T} 1$ tumors harvested from mice when tumor volume reached around $70 \mathrm{~mm}^{3}$. Total DNA and RNA were isolated from tumors using the AllPrep DNA/RNA Mini Kit (Qiagen), according to the manufacturer's instructions. Somatic variants were identified as described above and filtered before neoantigen prediction for normal tissue VAF (<2\%), tumor DNA and RNA VAF ( $>10 \%)$, and tumor RNA variant coverage ( $>5$ reads). Neoantigen prediction was performed on variants that passed filtering of both in vitro and in vivo data sets. The NetMHC algorithm (http://www.cbs.dtu.dk/services/ NetMHC/) was used to predict MHC-I binding for all possible 8- to 11-amino acid-long variants and corresponding WT peptides spanning the mutation using the pVAC-Seq pipeline $(56,57)$, with a cutoff of less than $300 \mathrm{nM}$ for the predicted affinity. Additionally, MHCII prediction was performed for mutated genes of interest (Adamts9 and Adgrf5) using the NetMHCII algorithm.
Raw reads of WES and RNA-Seq data for in vitro and in vivo data sets are available in the NCBI's Sequence Read Archive (SRA) (accession no. PRJNA596248).

MHC-I in vitro binding assay. Peptide binding to $\mathrm{H} 2-\mathrm{K}^{\mathrm{d}}$ or $\mathrm{H} 2-\mathrm{L}^{\mathrm{d}}$ was measured by determination of the stabilization of MHC-I molecules on RMA-S- $\mathrm{K}^{\mathrm{d}}$ and RMA-S- $\mathrm{L}^{\mathrm{d}}$ cells, respectively. RMA-S cells were incubated overnight at $26^{\circ} \mathrm{C}$ to promote the expression of empty MHC-I molecules at the cell surface (58). For the binding assay, $2 \times 10^{5}$ cells were incubated with peptides $(50$ or $100 \mu \mathrm{M})$ for 1 hour at $26^{\circ} \mathrm{C}$ followed by 2 hours at $37^{\circ} \mathrm{C}$. For the stability assay, cells were incubated with $50 \mu \mathrm{M}$ peptide at $26^{\circ} \mathrm{C}$ for 1 hour, then transferred at $37^{\circ} \mathrm{C}$ for 2,4 , or 6 hours. Cells were then washed with FACS buffer, and cell surface expression of $\mathrm{H} 2-\mathrm{K}^{\mathrm{d}}$ or $\mathrm{H} 2-\mathrm{L}^{\mathrm{d}}$ molecules was detected by flow cytometry using anti-H2- $\mathrm{K}^{\mathrm{d}}$ (clone SF1-1.1, BioLegend) or anti-H2- $\mathrm{L}^{\mathrm{d}}$ (30-5-7S, Thermo Fisher Scientific). Results are represented either as the mean fluorescence intensity (MFI) for $\mathrm{H} 2-\mathrm{K}^{\mathrm{d}}$ or $\mathrm{H} 2-\mathrm{L}^{\mathrm{d}}$ or as normalized binding, which was determined as MFI ratio (MFI observed in the presence of peptide/MFI observed in the absence of peptide). As a positive control for H2-L ${ }^{\mathrm{d}}$ binding, AH1-A5 (SPSYAYHQF), an optimized peptide ligand with better affinity for $\mathrm{H} 2-\mathrm{L}^{\mathrm{d}}$ than native $\mathrm{AH} 1$ (23) derived from envelope protein (gp70) of an endogenous ecotropic murine leukemia provirus, was used (59). The influenza epitope HA515 (IYSTVASSL) was used as positive control for $\mathrm{H} 2-\mathrm{K}^{\mathrm{d}}$ binding (60).

Vaccination procedure. Five- to six-week-old WT female BALB/c mice were obtained from Taconic. All mice were maintained under pathogen-free conditions in the animal facility at Weill Cornell Med- 
A

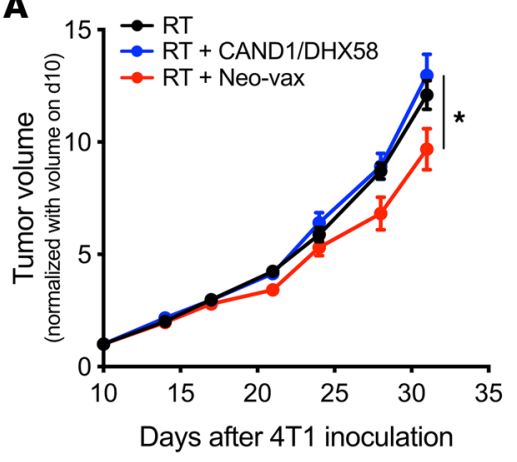

B

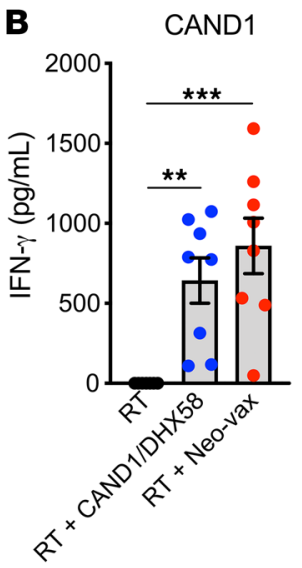

DHX58

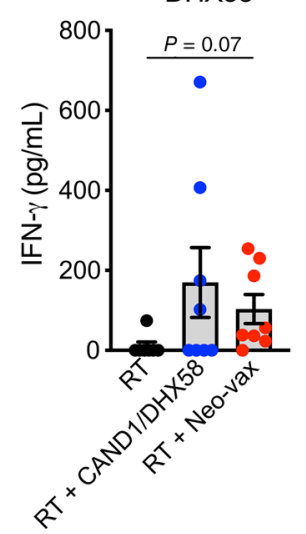

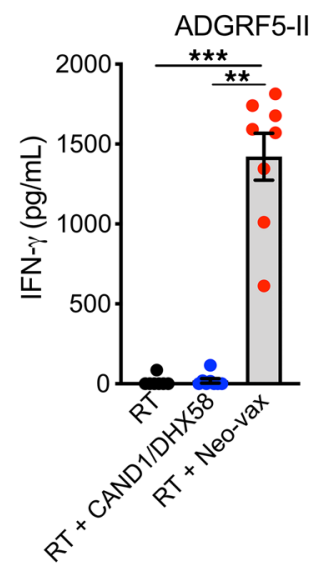

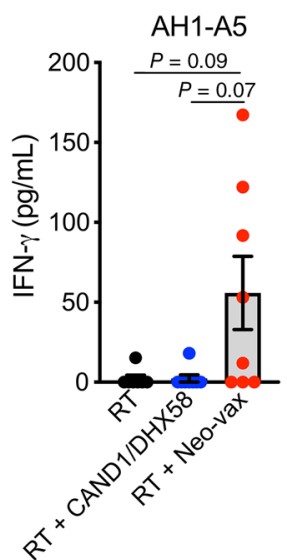

C

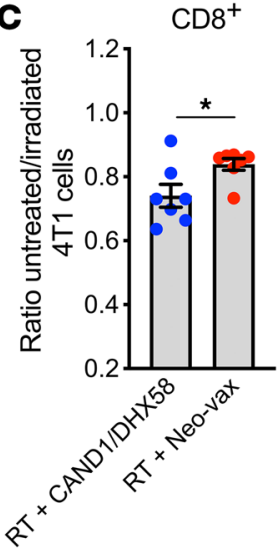

$\mathrm{CD}^{+}$

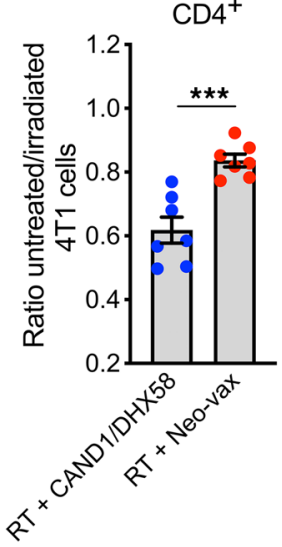

D

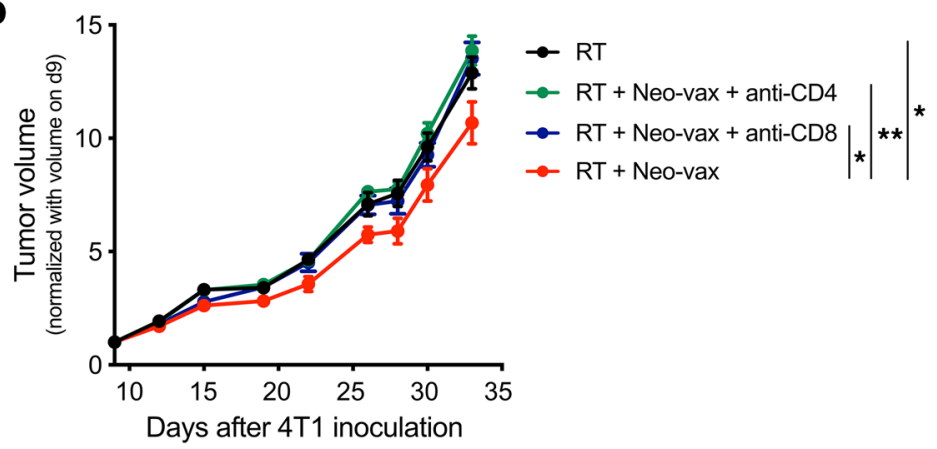

Figure 8. Both $\mathrm{CD4}^{+}$and $\mathrm{CD8}^{+}$neoantigen-specific $\mathrm{T}$ cells are required for the antitumor activity of the vaccine. (A) Mice were vaccinated weekly with adjuvant alone (RT), all 3 neoepitopes (Neo-vax), or only 2 neoepitopes (CAND1/DHX58) starting 2 weeks before 4T1 cell inoculation. Tumors were irradiated with a dose of 8 Gy on days 12,13 , and 14. Tumor volume over time; ${ }^{*} P<0.05$, with repeated-measures 2-way ANOVA ( $n=8-9$ mice per group). (B) Secreted IFN- $\gamma$ was measured in the supernatant of tumor-draining lymph node cells harvested on day 31 and stimulated 48 hours with indicated peptides (CAND1, DHX58, ADGRF5-II, and AH1-A5). ${ }^{* *} P<0.01,{ }^{* *} P<0.001$, with Kruskal-Wallis and Dunn's multiple-comparison tests $(n=8)$. (C) In vitro killing of untreated or irradiated 4T1 target cells (as described in Figure $3 \mathrm{E}$ ) using as effectors splenocytes harvested on day 31 and restimulated for 6 days with CAND1 or ADGRF5-II, followed by CD8 ${ }^{+}$or CD4 ${ }^{+} T$ cell isolation, respectively. Ratios of untreated to irradiated cells were calculated as described in Methods. ${ }^{*} P<0.05$, ${ }^{* *} P<0.001$, with unpaired 2-tailed Student's $t$ test $(n=7)$. (D) Tumor volume over time of mice $(n=7-8$ per group) treated as in $\mathbf{A}$ with adjuvant alone (RT) or with Neo-vax and RT. Some mice received CD4- or CD8-depleting antibodies starting 2 days before RT and maintained weekly; ${ }^{*} P<0.05$, ${ }^{*} P<0.01$, with repeated-measures 2-way ANOVA. All data presented are expressed as mean \pm SEM.

icine, and all experiments were approved by the Institutional Animal Care and Use Committee (IACUC) of the institution. Mice were anesthetized with isoflurane and immunized both in the footpad $(50 \mu \mathrm{L})$ and in the lower part of the leg $(100 \mu \mathrm{L})$ with the following vaccine solution (150 $\mu \mathrm{L}$ per mouse): poly(I:C) $(25 \mu \mathrm{g}), \mathrm{CpG}(10 \mu \mathrm{g})$, and 75 $\mu \mathrm{g}$ of each peptide, diluted in PBS (61). For the mice vaccinated with adjuvant only, DMSO was used at the same final concentration as the peptide-containing solutions.

$T$ cell restimulation assay. One week after the last vaccination, spleens and vaccine-draining lymph nodes (popliteal and inguinal) of immunized mice were harvested, processed into single-cell suspensions, and filtered using $40 \mu \mathrm{m}$ cell strainers. Red Blood Cell Lysis Buffer (catalog 00-4333-57, Thermo Fisher Scientific) was used to deplete erythrocytes from the splenic cell suspensions. Cells from lymph nodes were cultured in 96-well U-bottom plates overnight (about 14 hours) in complete T cell medium with indicated peptides $(2 \mu \mathrm{M}$ final $)$ in the presence of brefeldin A (catalog 00-4506-51, Thermo Fisher Scientific). H2-Kºinding
HA515 or H2-L ${ }^{\mathrm{d}}$-binding pMCMV (YPHFMPTNL; ref. 62) was used as irrelevant peptide as appropriate. Splenocytes were cultured in 24-well plates with indicated peptides ( $2 \mu \mathrm{M}$ final) for 5 days. On day 5 , irradiated feeder cells from naive BALB/c mice were pulsed with peptides and added to the splenocytes in culture, in addition to brefeldin A. After overnight stimulation, cells from lymph nodes or spleens were harvested, washed, and stained with Fixable Viability Dye eFluor 450 (Thermo Fisher Scientific). Cell surface staining was performed using the following anti-mouse antibodies (purchased from BioLegend): PE-conjugated CD8a (clone 53-6-7), PerCP/cyanine 5.5-conjugated CD4 (clone GK1.5), and APC-conjugated CD3e (clone 17A2). Cells were then fixed using IC Fixation buffer (catalog 00-8222, Thermo Fisher Scientific) and permeabilized using Permeabilization Buffer (catalog 00-8333, Thermo Fisher Scientific) before addition of the following antibodies: FITC-conjugated IFN- $\gamma$ (clone XMG1.2, BioLegend), APC/Cy7-conjugated TNF- $\alpha$ (clone MP6-XT22, BioLegend), PE-conjugated IL-4 (clone 11B11, BioLegend), PE-conjugated IL-5 (clone TRFK5, BioLegend), APC/Cy7-conjugat- 
A
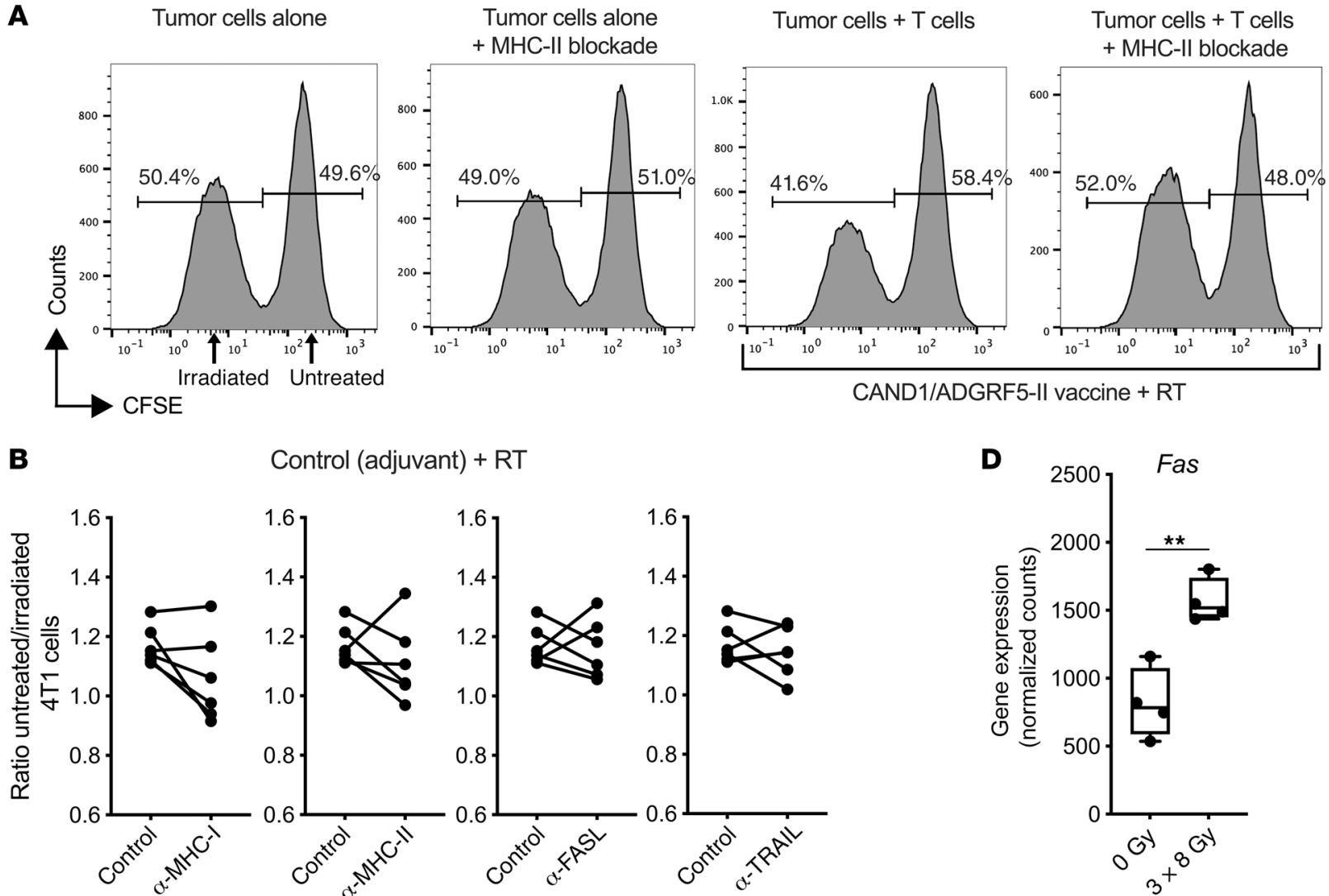

Control (adjuvant) + RT

C
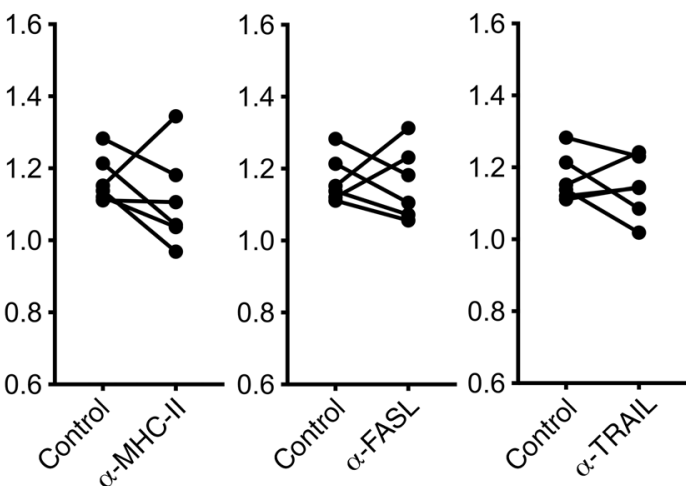

D
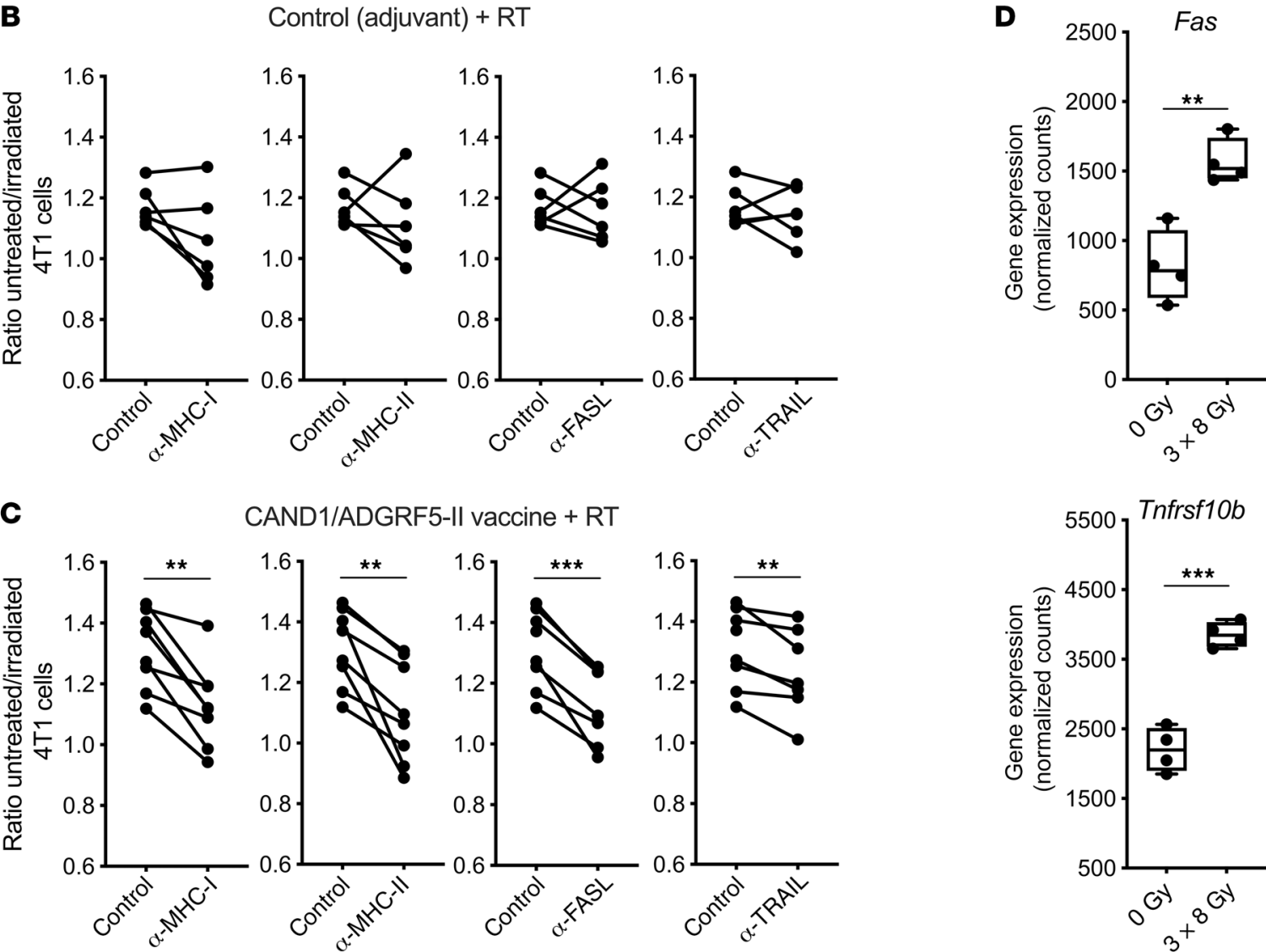

CAND1/ADGRF5-II vaccine + RT
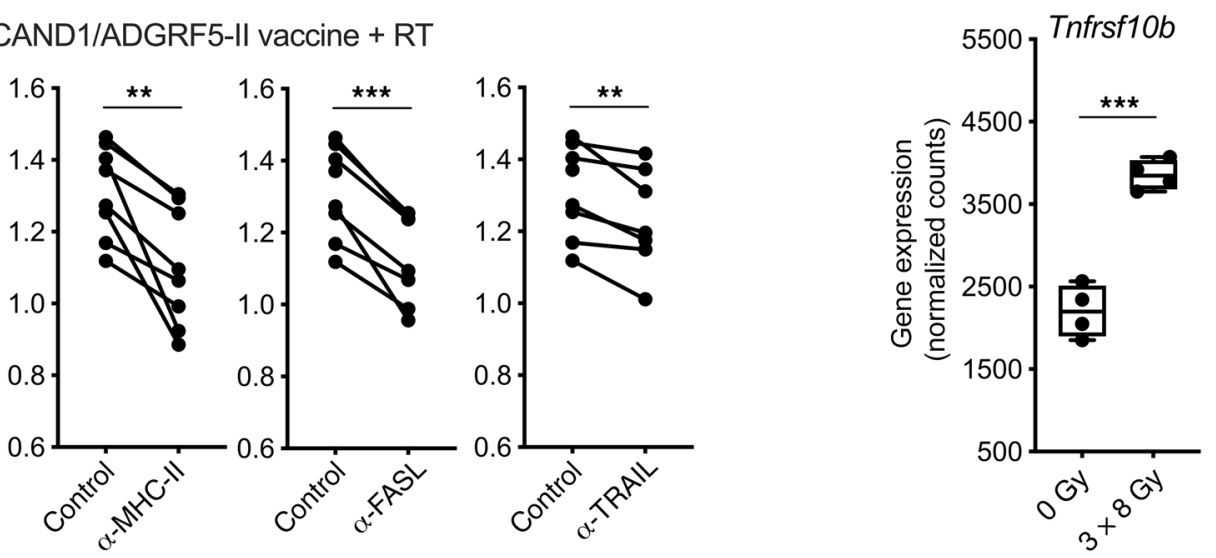

Figure 9. CD4+ and CD8+ neoantigen-specific T cells kill tumor cells in an MHC- and death receptor-dependent manner. (A-C) Mice were vaccinated with adjuvant alone or with CAND1/ADGRF5-II weekly, starting 2 weeks before 4T1 cell inoculation. Tumors were irradiated with a dose of 8 Gy on days 12 , 13, and 14. T cells were isolated from tumor-draining lymph nodes on day 20 and tested in an in vitro killing assay against untreated or irradiated 4T1 target cells (as described in Figure 3E), in the presence of blocking antibodies against MHC-I, MHC-II, FASL, and TRAIL. (A) Representative flow cytometry plots showing the 2 populations of $4 \mathrm{~T} 1$ cells. (B and $\mathbf{C}$ ) Ratios of untreated to irradiated cells calculated using absolute cell counts, as described in Methods. ${ }^{* *} P$ $<0.01$, ${ }^{* *} P<0.001$, with paired 2-tailed Student's $t$ test $(n=6-7)$. (D) Fas and Tnfrsf10b gene expression determined by RNA sequencing of 4T1 cells irradiated ( $3 \times 8$ Gy) or not ( 0 Gy) in vitro ( $n=4$ independent experiments). ${ }^{* *} P<0.01,{ }^{* *} P<0.001$, with unpaired 2 -tailed Welch's $t$ test. All data presented are expressed as mean \pm SEM.

ed IL-10 (clone JES5-16E3, BioLegend), and PE/Cy7-conjugated IL-13 (clone eBio13A, Thermo Fisher Scientific). Samples were acquired on the MACSQuant (Miltenyi Biotec) flow cytometer, and data were analyzed using FlowJo v10 software (BD Life Sciences). Cell culture supernatants from vaccine-draining lymph nodes were also collected after 48 hours of restimulation and stored at $-20^{\circ} \mathrm{C}$. Secreted IFN- $\gamma$ was then measured using the Quantikine ELISA IFN- $\gamma$ kit (catalog MIF100, R\&D Systems).
In vivo cytotoxicity assay. Antigen-specific cytotoxicity of $\mathrm{T}$ cells in vivo was evaluated as previously described $(26,27)$. Briefly, splenocytes from naive BALB/c mice were loaded with $10 \mu \mathrm{M}$ of neoepitopes (DHX58, CAND1, ADGRF5-II) or with $10 \mu \mathrm{M}$ of HA515 peptide for 2 hours at $37^{\circ} \mathrm{C}$, or were incubated without any peptides. Peptide-pulsed splenocytes were then labeled with $5 \mu \mathrm{M}$ CFSE (CFSE ${ }^{\text {hi }}$ ) (catalog 423801, BioLegend), while unpulsed splenocytes were labeled with 
$0.5 \mu \mathrm{M}$ CFSE $\left(\mathrm{CFSE}^{\mathrm{lo}}\right)$. One week after the second vaccination, a 50:50 mixture of $\mathrm{CFSE}^{\text {hi }}$ and $\mathrm{CFSE}^{\text {lo }}$ cells (1 million cells in $100 \mu \mathrm{L}$ ) were injected i.v. into the tail vein. Twenty-four hours later, spleens and inguinal lymph nodes were harvested. Single-cell suspensions were prepared, and cells were stained with APC-conjugated CD19 (clone eBio1D3, Thermo Fisher Scientific). Viability was determined using DAPI (D9542, MilliporeSigma). Samples were analyzed using the MACSQuant or the Fortessa (BD Biosciences) flow cytometer. Percentage of cytotoxicity was calculated using the following formula:

cytotoxicity $=100 \times\left[1-\left(\frac{\text { unloaded }}{\text { loaded }}\right)^{\text {control }} /\left(\frac{\text { unloaded }}{\text { loaded }}\right)^{\text {experimental }}\right]$

Equation 1

where "control" refers to the average ratio (unloaded/loaded) of target cells from the mice vaccinated with adjuvant alone.

In vitro cytotoxicity assay. One week after the second vaccination, vaccine-draining lymph nodes were harvested and processed into single-cell suspensions for coculture with CFSE-labeled target cells prepared as follows: Splenocytes from naive BALB/c mice were loaded with $10 \mu \mathrm{M}$ of neoepitopes or with WT peptides for 2 hours at $37^{\circ} \mathrm{C}$ and then labeled with CFSE. Neoepitope-pulsed splenocytes (CFSE ${ }^{\text {hi }}$ ) and WT peptide-pulsed splenocytes (CFSE ${ }^{10}$ ) were mixed 50:50 and cocultured overnight with effector cells at a ratio of 1:20. As a negative control, HA515-pulsed CFSE ${ }^{\text {hi }}$ cells were mixed with unpulsed CFSE ${ }^{\text {lo }}$ cells. Blocking of MHC-I was performed using anti- $\mathrm{K}^{\mathrm{d}}$ antibody (clone SF1.1.10, Bio X Cell) at a $10 \mu \mathrm{g} / \mathrm{mL}$ final concentration. After coculture, cells were stained for CD19 and viability and analyzed as described above for the in vivo cytotoxicity assay. The ratio of WT peptide-loaded to neoepitope-loaded target cells was used to calculate percentages of cytotoxicity. To determine in vitro killing of cancer cells, untreated $4 \mathrm{~T} 1$ cells were loaded with $5 \mu \mathrm{M}$ CFSE, whereas irradiated $(3 \times 8$ Gy) $4 \mathrm{~T} 1$ cells were loaded with $0.25 \mu \mathrm{M}$ CFSE, followed by coculture with effector cells for 12 hours at a 1:4 target/effector ratio. The ratio of untreated to irradiated $4 \mathrm{~T} 1$ cells was determined after gating on viable $\mathrm{CFSE}^{+}$cells, and the percentage of cytotoxicity toward irradiated cells was calculated using the following formula:

cytotoxicity $=100 \times\left[1-\left(\frac{\text { untreated }}{\text { irradiated }}\right)^{\text {control }} /\left(\frac{\text { untreated }}{\text { irradiated }}\right)^{\text {experimental }}\right]$

Equation 2

where "control" refers to the average ratio (untreated/irradiated) of target cells from the mice vaccinated with adjuvant alone. To determine the killing of tumor cells by $\mathrm{CD}^{+}$or $\mathrm{CD} 4^{+} \mathrm{T}$ cells, splenocytes from tumor-bearing mice were first cultured for 6 days with CAND1 or ADGRF5-II peptide, followed by isolation of $\mathrm{CD} 8^{+}$or $\mathrm{CD}^{+} \mathrm{T}$ cells, respectively, by negative selection (MicroBeads, Miltenyi Biotec). Coculture of T cells with CFSE-labeled 4T1 targets was performed as described above at a 1:2 target/effector ratio. For blocking experiments, T cells isolated (Pan T Cell Isolation Kit II, Mouse, Miltenyi Biotec) from lymph nodes of tumor-bearing mice were cultured with labeled $4 \mathrm{~T} 1$ cells at a 1:4 target/effector ratio for 12 hours, in the pres- ence of MHC-I-, MHC-II-, FASL-, or TRAIL-blocking antibody at a final concentration of $30 \mu \mathrm{g} / \mathrm{mL}$. In this last experiment, CountBright absolute counting beads (Thermo Fisher Scientific) were added to the samples before acquisition on the flow cytometer. The ratio of untreated to irradiated $4 \mathrm{~T} 1$ cells was determined after gating on viable $\mathrm{CFSE}^{+}$Epcam $^{+}$cells.

Tumor challenge and treatment. Mice were randomly assigned to treatment groups before tumor inoculation. One week after the second vaccination, mice were inoculated s.c. with $5 \times 10^{4} 4 \mathrm{~T} 1$ cells. Tumors were measured twice a week using a Vernier caliper, and volumes were calculated using the formula: length $\times$ width $^{2} \times \pi / 6$. On the first day of tumor measurement, i.e., day 8 to 10 after tumor inoculation, there were no statistically significant differences in mean tumor volume between mice vaccinated with adjuvant alone and those vaccinated with adjuvant plus peptides, but within each preassigned group, there was some variability in tumor size. Thus, tumor volume was normalized to the volume on the first day of measurement to more accurately determine the effect of radiation. Focal tumor radiotherapy was administered when the average tumor volume reached $60-80 \mathrm{~mm}^{3}$ in 3 doses of 8 Gy given on 3 consecutive days using the Small Animal Radiation Research Platform (SARRP; Xstrahl Ltd) as previously described (63). For the $\mathrm{T}$ cell depletion experiment, mice received i.p. injections of CD8a $(200 \mu \mathrm{g}), \mathrm{CD} 4(400 \mu \mathrm{g})$, or isotype control $(200 \mu \mathrm{g})$ antibodies on day 9 after tumor inoculation (i.e., 1 day before vaccination; 2 days before radiotherapy) and then repeated weekly. Depletion was confirmed to be greater than $80 \%$ in the tumor and greater than $95 \%$ in the spleen 6 days after injection of depleting antibodies by flow cytometry using noncompetitive clones: YTS156.7.7 (anti-CD8b) and RM4-4 (anti-CD4).

In the therapeutic experiment, mice were assigned to treatment groups (adjuvant + radiotherapy or Neo-vax + radiotherapy) on day 4 after tumor inoculation (when tumors were palpable). Vaccination started on day 4 and was repeated every 5-6 days thereafter. Mice were sacrificed when tumor exceeded $1.25 \mathrm{~cm}^{3}$ or if animals showed signs of distress per protocol.

Flow cytometry analysis of tumors and spleens. Tumors were enzymatically digested using a Mouse Tumor Dissociation Kit (catalog 130-096-730, Miltenyi Biotec) on a gentleMACS Octo Dissociator (Miltenyi Biotec). Following dissociation, cell suspensions were filtered through a $70 \mu \mathrm{m}$ strainer to remove large debris. Cell suspensions from the spleens were prepared as described above (see $T$ cell restimulation assay). Cells were stained for flow cytometry analysis using Fixable Viability Dye (eFluor 450 or eFluor 780). Fc receptors were then blocked using CD16/CD32 antibodies (clone 93, BioLegend) before surface staining with the following antibodies: v500-conjugated CD45 (clone 30-F11, BD Biosciences), FITC-conjugated CD8a (clone KT15, Thermo Fisher Scientific), Brilliant Violet 421conjugated CD3e (clone 17A2, BioLegend), PerCP/Cy5.5-conjugated CD4 (clone GK1.5, BioLegend), APC/Cy7-conjugated CD11b (clone M1/70, BioLegend), APC/Cy7-conjugated CD19 (clone 6D5, BioLegend), and Alexa Fluor 488-conjugated CD25 (clone PC61, BioLegend). Cells were then fixed and permeabilized using the eBioscience FOXP3 Transcription Factor kit (Thermo Fisher Scientific) followed by staining for intracellular targets using PE-conjugated FOXP3 (clone 150D/E4, Thermo Fisher Scientific) and PE/Cy7-conjugated T-bet (clone 4B10, BioLegend). CountBright absolute counting beads (Thermo Fisher Scientific) were added to the samples before acqui- 
sition on the MACSQuant flow cytometer, and data were analyzed using FlowJo v10 software.

For dextramer staining, cells were pretreated for 30 minutes at $37^{\circ} \mathrm{C}$ with dasatinib (50 nM; Sigma-Aldrich) before addition of the APC-conjugated CAND1-dextramer. Cells were stained for $30 \mathrm{~min}-$ utes at room temperature and washed, and the staining was pursued as described above. In this panel, the viability dye and the CD19/CD11b antibodies were used as the "dump" gate.

Lung metastasis quantification. To quantify spontaneous 4T1 clonogenic metastasis, lungs were removed and digested using the Mouse Lung Dissociation Kit (catalog 130-095-927, Miltenyi Biotec) on a gentleMACS Octo Dissociator. Cell suspensions were filtered through a $70 \mu \mathrm{m}$ strainer, washed, and resuspended in medium containing 60 $\mu \mathrm{M}$ 6-thioguanine (Sigma-Aldrich) and serially diluted in 6-well tissue culture plates $(43,44)$. After 11 days, plates were fixed with ethanol, and 6-thioguanine-resistant colonies were stained with $0.5 \%$ crystal violet (Sigma-Aldrich; diluted in $20 \%$ methanol) and counted. After accounting for dilution factors, data were expressed as total number of metastatic colonies per organ.

Targeted detection and quantification of MHC-I- and MHC-IIbound peptides. $4 \mathrm{~T} 1$ cells were irradiated with a dose of $8 \mathrm{~Gy}$ on 3 consecutive days $(3 \times 8 \mathrm{~Gy})$ or left untreated. Twenty-four hours after the third radiation dose, cells were harvested $\left(80 \times 10^{6}\right.$ cells $)$ and washed twice with cold PBS. Cell pellets were snap-frozen in liquid nitrogen and stored at $-80^{\circ} \mathrm{C}$ before cell lysis. Protein $\mathrm{G}$ magnetic beads conjugated to anti-MHC antibody (Omics Technologies Inc.) were added to the cell lysates at the ratio of $100 \times 10^{6}$ cells $/ 100 \mathrm{mg}$ beads. Immunoprecipitation was performed at $4^{\circ} \mathrm{C}$ for 15 hours on a rocking platform. Elution was performed according to Omics Technologies' protocol. Eluted peptide samples were cleaned 3 times in $9 \mathrm{M}$ urea using a $3 \mathrm{kDa}$ Amicon Filter (UFC501096, Millipore) and twice using Omics Technologies' proprietary washing buffer. The peptide solution was then acidified by addition of $1 \%$ trifluoroacetic acid (TFA) and incubated at room temperature for 15 minutes. A Sep-Pak light C18 cartridge (Waters Corp.) was activated by loading of $5 \mathrm{~mL} \mathrm{100 \%} \mathrm{(vol/vol)} \mathrm{ace-}$ tonitrile and was washed by $3.5 \mathrm{~mL} \mathrm{0.1 \%} \mathrm{TFA} \mathrm{solution} \mathrm{twice.} \mathrm{Acidi-}$ fied peptide solution was centrifuged at $1800 \mathrm{~g}$ for 5 minutes, and the supernatant was loaded into the cartridge. To further clean the peptides bound to the cartridge, $1 \mathrm{~mL}, 3 \mathrm{~mL}$, and $4 \mathrm{~mL}$ of $0.1 \%$ TFA were used sequentially. The peptides were eluted from the cartridge with $2 \mathrm{~mL}$ of $40 \%$ (vol/vol) acetonitrile with $0.1 \%$ TFA 3 times (for a total of $6 \mathrm{~mL}$ of eluate). The eluted peptides were lyophilized overnight, resuspended at $100 \mu \mathrm{g} / \mathrm{mL}$ in SCX solvent $\mathrm{A}\left(5 \mathrm{mM} \mathrm{KH}_{2} \mathrm{PO}_{4}\right.$ [pH 2.7], $30 \%$ acetonitrile), and fractionated by strong cation exchange chro-

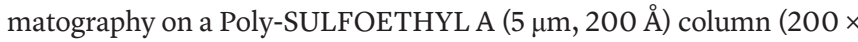
$9.4 \mathrm{~mm}$; PolyLC Inc.) using an increasing gradient of SCX solvent B (5 $\mathrm{mM} \mathrm{KH}_{2} \mathrm{PO}_{4}$ [pH 2.7], $30 \%$ acetonitrile, $350 \mathrm{mM} \mathrm{KCl}$ ) on an Agilent 1260 LC system. Collected fractions were subjected to an additional C18-based solid-phase extraction cleanup.

Transition parameters and retention times for each of the analyzed peptides were established individually using an Agilent 6495B Triple Quadrupole Mass Spectrometer for 2+ and 3+ charged precursor ions (Supplemental Table 1). Peptide samples were spiked with a mixture of internal controls composed by a pool of 10 -fmol heavy isotope-labeled peptides covering a large hydrophobicity window and a large $m / z$ range $(m / z 200-2500)$, and were subject to SRM analysis. The results were used to fine-tune the mass spectrometer for the best sensitivities around the molecular weights of the target peptides (Table 2). Peptide samples were eluted through an online Agilent 1290 HPLC system into the Jet Stream ESI source of an Agilent 6495B Triple Quadrupole Mass Spectrometer.

Data files containing the quantitative data of the relevant peptides were imported into Skyline 20.1. The abundance of a target peptide was represented by the total area under the curve (AUC) of all its optimized transitions normalized to the total AUC of the same transitions from heavy isotope-labeled peptide with the same sequence spiked in before the relevant peptide detection and quantification. Absolute quantification of each peptide was performed through application of AQUA Peptides (Sigma-Aldrich).

Statistics. Data are presented as mean \pm SEM. Statistical analysis was carried out as described in each corresponding figure legend, and sample sizes are shown in each corresponding figure legend. Where appropriate, $\log _{2}$ or arcsine square root transformation was applied to ensure the underlying model assumptions were satisfied. The Shapiro-Wilk normality test was then performed for all data sets to assess if a parametric or a nonparametric test was appropriate. Differences were considered statistically significant at $P$ less than 0.05 . For cytokine intracellular staining levels, difference across multiple experimental groups was examined using Kruskal-Wallis test, and pairwise differences were assessed using Dunn's test, which controls for familywise error rate in multiple comparisons. Differences in gene expression between untreated and irradiated samples were assessed using unpaired 2-tailed Welch's $t$ test on $\log _{2}$-transformed normalized counts. Differences in percentages of cytotoxicity between 2 "effector" groups were assessed using unpaired 2-tailed Welch's $t$ test, while comparisons between different "targets" were assessed using Kruskal-Wallis test and Dunn's tests. Differences in frequencies of cell populations in the tumors between treatment groups were calculated using Brown-Forsythe and Welch's ANOVA tests with Dunnett's T3 multiple-comparison test. When appropriate, overall differences due to Neo-vax or radiotherapy treatments were evaluated using 2-way ANOVA. The repeated-measures 2-way ANOVA test was used for tumor growth curve analyses. IFN- $\gamma$ measurements in the supernatants were compared using Kruskal-Wallis (or Mann-Whitney when 2 groups were compared) when the distribution of the values was not normal, or using 1-way ANOVA otherwise. Differences in mouse survival times were examined using log-rank test. All data were analyzed using GraphPad Prism version 8 software.

Study approval. All animal studies were approved by the IACUC of Weill Cornell Medicine (protocol 2015-0028).

\section{Author contributions}

CL designed and conducted the experiments, acquired and analyzed data, and wrote the manuscript. NPR performed analysis of whole-exome and RNA sequencing data with the help of TZ. CCC and LS aided in analysis of mass spectrometry data. NPR, TY, MC, and ND contributed to the performing of experimental work. LG and SCF reviewed the manuscript. XKZ supervised statistical analysis. SD conceived the study, reviewed the data, and wrote the manuscript. All authors approved the final version of the manuscript.

\section{Acknowledgments}

This work was supported by grants from the NIH (R01CA198533 and CA201246) to SD. CL is supported, in part, by the American 
Association for Cancer Research triple-negative breast cancer fellowship (18-40-43-LHUI). LG is supported by a Breakthrough Level 2 grant from the US Department of Defense, Breast Cancer Research Program (BC180476P1). SCF and SD are supported by a grant from the Breast Cancer Research Foundation (BCRF-19053). The mouse irradiator was purchased with the support of National Cancer Institute grant S10RR0267619. The schematic images in Figure $3 \mathrm{E}$ and the graphical abstract were created with BioRender (biorender.com). Recombinant human IL-2 was provided by the Biological Resources Branch, Developmental Ther- apeutics Program, Division of Cancer Treatment and Diagnosis, National Cancer Institute.

Address correspondence to: Sandra Demaria, Department of Radiation Oncology, Weill Cornell Medicine, 1300 York Ave., Rm E-215D, Box 169, New York, New York 10065, USA. Phone: 646.962.2092; Email: szd3005@med.cornell.edu.

NPR's present address is: The University of Texas MD Anderson Cancer Center, Houston, Texas, USA.
1. Schumacher TN, Schreiber RD. Neoantigens in cancer immunotherapy. Science. 2015;348(6230):69-74.

2. Rizvi NA, et al. Cancer immunology. Mutational landscape determines sensitivity to PD-1 blockade in non-small cell lung cancer. Science. 2015;348(6230):124-128.

3. Le DT, et al. Mismatch repair deficiency predicts response of solid tumors to $\mathrm{PD}-1$ blockade. Science. 2017;357(6349):409-413.

4. Snyder A, et al. Genetic basis for clinical response to CTLA-4 blockade in melanoma. $N$ Engl JMed. 2014;371(23):2189-2199.

5. Ott PA, et al. An immunogenic personal neoantigen vaccine for patients with melanoma. Nature. 2017;547(7662):217-221.

6. Keskin DB, et al. Neoantigen vaccine generates intratumoral $\mathrm{T}$ cell responses in phase Ib glioblastoma trial. Nature. 2019;565(7738):234-239.

7. Sahin U, et al. Personalized RNA mutanome vaccines mobilize poly-specific therapeutic immunity against cancer. Nature. 2017;547(7662):222-226.

8 . Hilf $\mathrm{N}$, et al. Actively personalized vaccination trial for newly diagnosed glioblastoma. Nature. 2019;565(7738):240-245.

9. Garcia-Garijo A, et al. Determinants for neoantigen identification. Front Immunol. 2019;10:1392.

10. Roudko V, et al. Computational prediction and validation of tumor-associated neoantigens. Front Immunol. 2020;11:27.

11. Castle JC, et al. Exploiting the mutanome for tumor vaccination. Cancer Res. 2012;72(5):1081-1091.

12. Duan F, et al. Genomic and bioinformatic profiling of mutational neoepitopes reveals new rules to predict anticancer immunogenicity. J Exp Med. 2014;211(11):2231-2248.

13. Bassani-Sternberg M, et al. Mass spectrometry of human leukocyte antigen class I peptidomes reveals strong effects of protein abundance and turnover on antigen presentation. Mol Cell Proteomics. 2015;14(3):658-673.

14. Yewdell JW, et al. Making sense of mass destruction: quantitating MHC class I antigen presentation. Nat Rev Immunol. 2003;3(12):952-961.

15. Knijnenburg TA, et al. Genomic and molecular landscape of DNA damage repair deficiency across The Cancer Genome Atlas. Cell Rep. 2018;23(1):239-254.

16. McLaughlin M, et al. Inflammatory microenvironment remodelling by tumour cells after radiotherapy. Nat Rev Cancer. 2020;20(4):203-217.

17. Reits EA, et al. Radiation modulates the peptide repertoire, enhances MHC class I expression, and induces successful antitumor immunothera- py. J Exp Med. 2006;203(5):1259-1271.

18. Harding SM, et al. Mitotic progression following DNA damage enables pattern recognition within micronuclei. Nature. 2017;548(7668):466-470.

19. Formenti SC, et al. Radiotherapy induces responses of lung cancer to CTLA-4 blockade. Nat Med. 2018;24(12):1845-1851.

20. Lhuillier C, et al. Radiation therapy and anti-tumor immunity: exposing immunogenic mutations to the immune system. Genome Med. 2019;11(1):40.

21. Dewan MZ, et al. Fractionated but not singledose radiotherapy induces an immunemediated abscopal effect when combined with anti-CTLA-4 antibody. Clin Cancer Res. 2009;15(17):5379-5388.

22. Theelen W, et al. Effect of pembrolizumab after stereotactic body radiotherapy vs pembrolizumab alone on tumor response in patients with advanced non-small cell lung cancer: results of the PEMBRO-RT phase 2 randomized clinical trial. JAMA Oncol. 2019;5(9):1276-1282.

23. Slansky JE, et al. Enhanced antigen-specific antitumor immunity with altered peptide ligands that stabilize the MHC-peptide-TCR complex. Immunity. 2000;13(4):529-538.

24. van der Burg SH, et al. Immunogenicity of peptides bound to MHC class I molecules depends on the MHC-peptide complex stability. J Immunol.1996;156(9):3308-3314.

25. Kreiter S, et al. Mutant MHC class II epitopes drive therapeutic immune responses to cancer. Nature. 2015;520(7549):692-696.

26. Nelson D, et al. In vivo cross-presentation of a soluble protein antigen: kinetics, distribution, and generation of effector CTL recognizing dominant and subdominant epitopes. JImmunol. 2000;165(11):6123-6132.

27. Quezada SA, et al. Tumor-reactive CD4(+) T cells develop cytotoxic activity and eradicate large established melanoma after transfer into lymphopenic hosts. JExp Med. 2010;207(3):637-650.

28. Marx V. Targeted proteomics. Nat Methods. 2013;10(1):19-22.

29. Alspach E, et al. MHC-II neoantigens shape tumour immunity and response to immunotherapy. Nature. 2019;574(7780):696-701.

30. Salomon N, et al. A liposomal RNA vaccine inducing neoantigen-specific $\mathrm{CD} 4^{+} \mathrm{T}$ cells augments the antitumor activity of local radiotherapy in mice. Oncoimmunology. 2020;9(1):1771925.

31. Kotov DI, et al. Many Th cell subsets have Fas Ligand-dependent cytotoxic potential. J Immunol. 2018;200(6):2004-2012.
32. Cristescu R, et al. Pan-tumor genomic biomarkers for PD-1 checkpoint blockade-based immunotherapy. Science. 2018;362(6411):eaar3593.

33. Yarchoan M, et al. Tumor mutational burden and response rate to PD-1 inhibition. $N$ Engl J Med. 2017;377(25):2500-2501.

34. Widau RC, et al. RIG-I-like receptor LGP2 protects tumor cells from ionizing radiation. Proc Natl Acad Sci U S A. 2014;111(4):E484-E491.

35. Gasser S, et al. The DNA damage pathway regulates innate immune system ligands of the NKG2D receptor. Nature. 2005;436(7054):1186-1190.

36. Kubo F, et al. Loss of the adhesion G-protein coupled receptor ADGRF5 in mice induces airway inflammation and the expression of CCL2 in lung endothelial cells. Respir Res. 2019;20(1):11.

37. Marty Pyke R, et al. Evolutionary pressure against MHC class II binding cancer mutations. Cell. 2018;175(2):416-428.

38. Linnemann C, et al. High-throughput epitope discovery reveals frequent recognition of neoantigens by CD4+ T cells in human melanoma. Nat Med. 2015;21(1):81-85.

39. Tran E, et al. Cancer immunotherapy based on mutation-specific CD4+ T cells in a patient with epithelial cancer. Science. 2014;344(6184):641-645.

40. Oh DY, et al. Intratumoral CD $4^{+} \mathrm{T}$ cells mediate anti-tumor cytotoxicity in human bladder cancer. Cell. 2020;181(7):1612-1625.

41. Zhang B, et al. Induced sensitization of tumor stroma leads to eradication of established cancer by T cells. J Exp Med. 2007;204(1):49-55.

42. Haabeth OAW, et al. CD4 ${ }^{+}$T-cell-mediated rejection of $\mathrm{MHC}$ class II-positive tumor cells is dependent on antigen secretion and indirect presentation on host APCs. Cancer Res. 2018;78(16):4573-4585.

43. Pulaski BA, et al. Immunotherapy with vaccines combining MHC class II/CD80+ tumor cells with interleukin-12 reduces established metastatic disease and stimulates immune effectors and monokine induced by interferon gamma. Cancer Immunol Immunother. 2000;49(1):34-45.

44. Pulaski BA, et al. Cooperativity of staphylococcal aureus enterotoxin B superantigen, major histocompatibility complex class II, and CD80 for immunotherapy of advanced spontaneous metastases in a clinically relevant postoperative mouse breast cancer model. Cancer Res. 2000;60(10):2710-2715.

45. Hu Z, et al. Towards personalized, tumourspecific, therapeutic vaccines for cancer. Nat Rev Immunol. 2018;18(3):168-182.

46. Aslakson CJ, Miller FR. Selective events in the 
metastatic process defined by analysis of the sequential dissemination of subpopulations of a mouse mammary tumor. Cancer Res. 1992;52(6):1399-1405.

47. Powis SJ, et al. Restoration of antigen presentation to the mutant cell line RMA-S by an MHC-linked transporter. Nature. 1991;354(6354):528-531.

48. Alexander-Miller MA, et al. Alloreactive cytotoxic $T$ lymphocytes generated in the presence of viral-derived peptides show exquisite peptide and MHC specificity. J Immunol. 1993;151(1):1-10.

49. Martin M. Cutadapt removes adapter sequences from high-throughput sequencing reads. EMBnetjournal. 2011;17(1):10-12.

50. Li H. Aligning sequence reads, clone sequences and assembly contigs with BWA-MEM [preprint]. https://arxiv.org/abs/1303.3997. Posted on arXiv March 16, 2013.

51. DePristo MA, et al. A framework for variation discovery and genotyping using nextgeneration DNA sequencing data. Nat Genet. 2011;43(5):491-498.
52. Koboldt DC, et al. VarScan 2: somatic mutation and copy number alteration discovery in cancer by exome sequencing. Genome Res. 2012;22(3):568-576.

53. Dobin A, et al. STAR: ultrafast universal RNA-seq aligner. Bioinformatics. 2013;29(1):15-21.

54. Anders S, et al. HTSeq-a Python framework to work with high-throughput sequencing data. Bioinformatics. 2015;31(2):166-169.

55. Love MI, et al. Moderated estimation of fold change and dispersion for RNA-seq data with DESeq2. Genome Biol. 2014;15(12):550.

56. Hundal J, et al. pVACtools: a computational toolkit to identify and visualize cancer neoantigens. Cancer Immunol Res. 2020;8(3):409-420.

57. Andreatta M, Nielsen M. Gapped sequence alignment using artificial neural networks: application to the MHC class I system. Bioinformatics. 2016;32(4):511-517.

58. Schumacher TN, et al. Direct binding of peptide to empty MHC class I molecules on intact cells and in vitro. Cell. 1990;62(3):563-567.
59. Huang AY, et al. The immunodominant major histocompatibility complex class I-restricted antigen of a murine colon tumor derives from an endogenous retroviral gene product. Proc Natl Acad Sci U S A. 1996;93(18):9730-9735.

60. Tamura M, et al. Definition of amino acid residues on the epitope responsible for recognition by influenza A virus $\mathrm{H} 1$-specific, $\mathrm{H} 2$-specific, and $\mathrm{H} 1$ - and H2-cross-reactive murine cytotoxic T-lymphocyte clones. J Virol. 1998;72(11):9404-9406.

61. Charlebois R, et al. PolyI:C and CpG synergize with anti-ErbB2 $\mathrm{mAb}$ for treatment of breast tumors resistant to immune checkpoint inhibitors. Cancer Res. 2017;77(2):312-319.

62. Del Val M, et al. Presentation of CMV immediate-early antigen to cytolytic T lymphocytes is selectively prevented by viral genes expressed in the early phase. Cell. 1989;58(2):305-315.

63. Wennerberg E, et al. CD73 blockade promotes dendritic cell infiltration of irradiated tumors and tumor rejection. Cancer Immunol Res. 2020;8(4):465-478. 